\title{
DERIVATION OF GINZBURG-LANDAU THEORY FOR A ONE-DIMENSIONAL SYSTEM WITH CONTACT INTERACTION
}

\author{
RUPERT L. FRANK, CHRISTIAN HAINZL, ROBERT SEIRINGER, \\ AND JAN PHILIP SOLOVEJ
}

\begin{abstract}
In a recent paper [7] we give the first rigorous derivation of the celebrated Ginzburg-Landau (GL) theory, starting from the microscopic BardeenCooper-Schrieffer (BCS) model. Here we present our results in the simplified case of a one-dimensional system of particles interacting via a $\delta$-potential.
\end{abstract}

\section{Introduction and Main Results}

1.1. Introduction. In 1950 Ginzburg and Landau [1 presented the first satisfactory mathematical description of the phenomenon of superconductivity. Their model examined the macroscopic properties of a superconductor in a phenomenological way, without explaining its microscopic mechanism. In the GL theory the superconducting state is represented by a complex order parameter $\psi(x)$, which is zero in the normal state and non-zero in the superconducting state. The order parameter $\psi(x)$ can be considered as a macroscopic wave-function whose square $|\psi(x)|^{2}$ is proportional to the density of superconducting particles.

In 1957 Bardeen, Cooper and Schrieffer [2 formulated the first microscopic explanation of superconductivity starting from a first principle Hamiltonian. In a major breakthrough they realized that this phenomenon can be described by the pairing-mechanism. The superconducting state forms due to an instability of the normal state in the presence of an attraction between the particles. In the case of a metal the attraction is made possible by an interaction through the lattice. For other systems, like superfluid cold gases, the interaction is of local type. In the BCS theory the superconducting state, which is made up by pairs of particles of opposite spin, the Cooper-pairs, is described by a two-particle wave-function $\alpha(x, y)$.

A connection between the two approaches, the phenomenological GL theory and the microscopic BCS theory, was made by Gorkov [3] who showed that, close to the critical temperature, the order parameter $\psi(x)$ and the pair-wavefunction $\alpha(x, y)$ are proportional. A simpler argument was later given by de Gennes [4.

Recently we presented in [7] a mathematical proof of the equivalence of the two models, GL and BCS, in the limit when the temperature $T$ is close to the critical temperature $T_{c}$, i.e., when $h=\left[\left(T_{c}-T\right) / T_{c}\right]^{1 / 2} \ll 1$, where $T_{c}$ is the critical temperature for the translation-invariant BCS equation. The mathematical aspects of this equation where studied in detail in [8, 6, 9, 10, 11]. In the present paper we present this result in the simplified case a of one-dimensional system where the

Date: March 9, 2011. 
particles interact via an attractive contact interaction potential of the form

$$
V(x-y)=-a \delta(x-y) \quad \text { with } a>0 .
$$

We assume that the system is subject to a weak external potential $W$, which varies on a large scale $1 / h$ compared to the microscopic scale of order 1 . Since variations of the system on the macroscopic scale cause a change in energy of the order $h^{2}$, we assume that the external potential $W$ is also of the order $h^{2}$. Hence we write it as $h^{2} W(h x)$, with $x$ being the microscopic variable. The parameter $h$ will play the role of a semiclassical parameter.

We will prove that, to leading order in $h$, the Cooper pair wave function $\alpha(x, y)$ and the GL function $\psi(x)$ are related by

$$
\alpha(x, y)=\psi\left(h \frac{x+y}{2}\right) \alpha^{0}(x-y)
$$

where $\alpha^{0}$ is the translation invariant minimizer of the BCS functional. In particular, the argument $\bar{x}$ of the order parameter $\psi(\bar{x})$ describes the center-of-mass motion of the BCS state, which varies on the macroscopic scale. To be precise, we shall prove that $\alpha(x, y)=\frac{1}{2}(\psi(h x)+\psi(h y)) \alpha^{0}(x-y)$ to leading order in $h$, which agrees with (1.2) to this order.

For simplicity we restrict our attention to contact potentials of the form (1.1), but our method can be generalized to other kinds of interactions; see [7] for details. The proof presented here is simpler than the general proof in [7] which applies to any dimension $d \leq 3$. There are several reasons for this. First, there is no magnetic field in one dimension. Second, for a contact interaction the translation invariant problem is particularly simple and the corresponding gap equation has an explicit solution. Finally, several estimates are simpler in one dimension due the boundedness of the Green's function for the Laplacian.

1.2. The BCS Functional. We consider a macroscopic sample of a fermionic system, in one spatial dimension. Let $\mu \in \mathbb{R}$ denote the chemical potential and $T>0$ the temperature of the sample. The fermions interact through the attractive two-body potential given in (1.1). In addition, they are subject to an external force, represented by a potential $W(x)$.

In BCS theory the state of the system can be conveniently described in terms of a $2 \times 2$ operator valued matrix

$$
\Gamma=\left(\begin{array}{cc}
\gamma & \alpha \\
\bar{\alpha} & 1-\bar{\gamma}
\end{array}\right)
$$

satisfying $0 \leq \Gamma \leq 1$ as an operator on $L^{2}(\mathbb{R}) \oplus L^{2}(\mathbb{R})$. The bar denotes complex conjugation, i.e., $\bar{\alpha}$ has the integral kernel $\overline{\alpha(x, y)}$. In particular, $\Gamma$ is assumed to be hermitian, which implies that $\gamma$ is hermitian and $\alpha$ is symmetric (i.e, $\gamma(x, y)=$ $\overline{\gamma(y, x)}$ and $\alpha(x, y)=\alpha(y, x)$.) There are no spin variables in $\Gamma$. The full, spin dependent Cooper pair wave function is the product of $\alpha$ with an antisymmetric spin singlet.

We are interested in the effect of weak and slowly varying external fields, described by a potential $h^{2} W(h x)$. In order to avoid having to introduce boundary conditions, we assume that the system is infinite and periodic with period $h^{-1}$. In particular, $W$ should be periodic. We also assume that the state $\Gamma$ is periodic. The aim then is to calculate the free energy per unit volume. 
We find it convenient to do a rescaling and use macroscopic variables instead of the microscopic ones. In macroscopic variables, the BCS functional has the form

$$
\mathcal{F}^{\mathrm{BCS}}(\Gamma):=\operatorname{Tr}\left(-h^{2} \nabla^{2}-\mu+h^{2} W(x)\right) \gamma-T S(\Gamma)-a h \int_{\mathcal{C}}|\alpha(x, x)|^{2} d x
$$

where $\mathcal{C}$ denotes the unit interval $[0,1]$. The entropy equals $S(\Gamma)=-\operatorname{Tr} \Gamma \ln \Gamma$. The BCS state of the system is a minimizer of this functional over all admissible $\Gamma$.

The symbol Tr in (1.3) stands for the trace per unit volume. More precisely, if $B$ is a periodic operator (meaning that it commutes with translation by 1 ), then $\operatorname{Tr} B$ equals, by definition, the (usual) trace of $\chi B$, with $\chi$ the characteristic function of $\mathcal{C}$. The location of the interval is obviously of no importance. It is not difficult to see that the trace per unit volume has the usual properties like cyclicity, and standard inequalities like Hölder's inequality hold. This is discussed in more detail in 7 .

Assumption 1. We assume that $W$ is a bounded, periodic function with period 1 and $\int_{\mathcal{C}} W(x) d x=0$.

1.2.1. The Translation-Invariant Case. In the translation invariant case $W=0$ one can restrict $\mathcal{F}^{\mathrm{BCS}}$ to translation invariant states. We write a general translation invariant state in form of the $2 \times 2$ matrix

$$
\Gamma=\left(\begin{array}{cc}
\frac{\tilde{\gamma}(-i h \nabla)}{\tilde{\alpha}(-i h \nabla)} & 1-\frac{\tilde{\alpha}(-i h \nabla)}{\tilde{\gamma}(-i h \nabla)}
\end{array}\right),
$$

that is, $\gamma=[\Gamma]_{11}$ and $\alpha=[\Gamma]_{12}$ have integral kernels

$$
\gamma(x, y)=\frac{1}{2 \pi} \int_{\mathbb{R}} \tilde{\gamma}(h p) e^{i p(x-y)} d p \quad \text { and } \quad \alpha(x, y)=\frac{1}{2 \pi} \int_{\mathbb{R}} \tilde{\alpha}(h p) e^{i p(x-y)} d p .
$$

The fact that $\Gamma$ is admissible means that $\tilde{\alpha}(p)=\overline{\tilde{\alpha}(-p)}$, that $0 \leq \tilde{\gamma}(p) \leq 1$ and $|\tilde{\alpha}(p)|^{2} \leq \tilde{\gamma}(p)(1-\tilde{\gamma}(-p))$ for any $p \in \mathbb{R}$. For states of this form the BCS functional becomes

$$
\mathcal{F}^{\mathrm{BCS}}(\Gamma)=\int_{\mathbb{R}}\left(h^{2} p^{2}-\mu\right) \tilde{\gamma}(h p) \frac{d p}{2 \pi}-T \int_{\mathbb{R}} S(\tilde{\Gamma}(h p)) \frac{d p}{2 \pi}-a h\left|\int_{\mathbb{R}} \tilde{\alpha}(h p) \frac{d p}{2 \pi}\right|^{2},
$$

with $S(\tilde{\Gamma}(p))=-\operatorname{Tr}_{\mathbb{C}^{2}} \tilde{\Gamma}(p) \ln \tilde{\Gamma}(p)$ and $\tilde{\Gamma}(p)$ the $2 \times 2$ matrix obtained by replacing $-i \nabla$ by $p$ in (1.4).

In the following, we are going to summarize some well-known facts about the translation invariant functional (1.5). For given $a>0$, we define the critical temperature $T_{c}>0$ by the equation

$$
\frac{1}{a}=\int_{\mathbb{R}} \frac{\tanh \left(\frac{p^{2}-\mu}{2 T_{c}}\right)}{p^{2}-\mu} \frac{d p}{2 \pi}
$$

The fact that there is a unique solution to this equation follows from the strict monotonicity of $t / \tanh t$ for $t>0$. If $T \geq T_{c}$, then the minimizer of (1.5) satisfies $\tilde{\alpha} \equiv 0$ and $\tilde{\gamma}(h p)=\left(1+\exp \left(\left(h^{2} p^{2}-\mu\right) / T\right)\right)^{-1}$. If $0<T<T_{c}$, on the other hand, then there is a unique solution $\Delta_{0}>0$ of the BCS gap equation

$$
\frac{1}{a}=\int_{\mathbb{R}} \frac{1}{K_{T}^{0}(p)} \frac{d p}{2 \pi}
$$


where

$$
K_{T}^{0}(p)=\frac{\sqrt{\left(p^{2}-\mu\right)^{2}+\Delta_{0}^{2}}}{\tanh \left(\frac{1}{2 T} \sqrt{\left(p^{2}-\mu\right)^{2}+\Delta_{0}^{2}}\right)} .
$$

Moreover, the minimizer of (1.5) is given by

$$
\tilde{\Gamma}^{0}(h p)=\left(1+\exp \left(\frac{1}{T} H_{\Delta_{0}}^{0}(h p)\right)\right)^{-1}
$$

with

$$
H_{\Delta_{0}}^{0}(p)=\left(\begin{array}{cc}
p^{2}-\mu & -\Delta_{0} \\
-\Delta_{0} & -p^{2}+\mu
\end{array}\right) .
$$

Writing $\tilde{\alpha}^{0}(h p)=\left[\tilde{\Gamma}^{0}(h p)\right]_{12}$ one easily deduces from (1.9) that

$$
\tilde{\alpha}^{0}(p)=\frac{\Delta_{0}}{2 K_{T}^{0}(p)} .
$$

To summarize, in the case $W \equiv 0$ the functional $\mathcal{F}^{\mathrm{BCS}}$ has a minimizer $\Gamma^{0}$ for $0<T<T_{c}$ whose off-diagonal element does not vanish and has the integral kernel

$$
\alpha^{0}((x-y) / h)=\frac{\Delta_{0}}{2} \int_{\mathbb{R}} \frac{1}{K_{T}^{0}(h p)} e^{i p(x-y)} \frac{d p}{2 \pi} .
$$

We emphasize that the function $\alpha^{0}$ depends on $T$. For $T$ close to $T_{c}$, which is the case of interest, we have $\Delta_{0} \sim \operatorname{const}\left(1-T / T_{c}\right)^{1 / 2}$.

1.3. The GL Functional. Let $\psi$ be a periodic function in $H_{\text {loc }}^{1}(\mathbb{R})$. For numbers $b_{1}, b_{3}>0$ and $b_{2} \in \mathbb{R}$ the Ginzburg-Landau (GL) functional is given by

$$
\mathcal{E}(\psi)=\int_{\mathcal{C}}\left(b_{1}\left|\psi^{\prime}(x)\right|^{2}+b_{2} W(x)|\psi(x)|^{2}+b_{3}\left(1-|\psi(x)|^{2}\right)^{2}\right) d x .
$$

We denote its ground state energy by

$$
E^{\mathrm{GL}}=\inf \left\{\mathcal{E}(\psi) \mid \psi \in H_{\text {per }}^{1}\right\} .
$$

Under our assumptions on $W$ it is not difficult to show that there is a corresponding minimizer, which satisfies a second order differential equation known as the GL equation.

1.4. Main Results. Recall the definition of the BCS functional $\mathcal{F}^{\mathrm{BCS}}$ in (1.3). We define the energy $F^{\mathrm{BCS}}(T, \mu)$ as the difference between the infimum of $\mathcal{F}^{\mathrm{BCS}}$ over all admissible $\Gamma$ and the free energy of the normal state

$$
\Gamma_{0}:=\left(\begin{array}{cc}
\gamma_{0} & 0 \\
0 & 1-\bar{\gamma}_{0}
\end{array}\right)
$$

with $\gamma_{0}=\left(1+e^{\left(-h^{2} \nabla^{2}+h^{2} W(x)-\mu\right) / T}\right)^{-1}$. That is,

$$
F^{\mathrm{BCS}}(T, \mu)=\inf _{\Gamma} \mathcal{F}^{\mathrm{BCS}}(\Gamma)-\mathcal{F}^{\mathrm{BCS}}\left(\Gamma_{0}\right) .
$$

Note that

$$
\mathcal{F}^{\mathrm{BCS}}\left(\Gamma_{0}\right)=-T \operatorname{Tr} \ln \left(1+\exp \left(-\left(-h^{2} \nabla^{2}-\mu+h^{2} W(x)\right)\right) / T\right) .
$$

For small $h$ this behaves like an (explicit) constant times $h^{-1}$. Under further regularity assumptions on $W$, 1.15) can be expanded in powers of $h$. We do not need this, however, since we are only interested in the difference $F^{\mathrm{BCS}}(T, \mu)$. 
Since $\Gamma_{0}$ is an admissible state, one always has $F^{\mathrm{BCS}}(T, \mu) \leq 0$. If the strict inequality $F^{\mathrm{BCS}}(T, \mu)<0$ holds, then the system is said to be in a superconducting (or superfluid, depending on the physical interpretation) state.

THEOREM 1. Let Assumption [1] be satisfied, and let $T_{c}>0$ be the critical temperature in the translation invariant case, defined in (1.6). Let $D>0$. Then there are coefficients $b_{1}, b_{2}$ and $b_{3}$, given explicitly in (1.20)-(1.22) below, such that

$$
F^{\mathrm{BCS}}\left(T_{c}\left(1-D h^{2}\right), \mu\right)=h^{3}\left(E^{\mathrm{GL}}-b_{3}\right)+o\left(h^{3}\right)
$$

as $h \rightarrow 0$. More precisely, the error term o $\left(h^{3}\right)$ satisfies

$$
- \text { const } h^{3+\frac{1}{3}} \leq o\left(h^{3}\right) \leq \text { const } h^{5} .
$$

Moreover, if $\Gamma$ is an approximate minimizer of $\mathcal{F}^{\mathrm{BCS}}$ at $T=T_{c}\left(1-h^{2} D\right)$, in the sense that

$$
\mathcal{F}^{\mathrm{BCS}}(\Gamma) \leq \mathcal{F}^{\mathrm{BCS}}\left(\Gamma_{0}\right)+h^{3}\left(E^{\mathrm{GL}}-b_{3}+\epsilon\right)
$$

for some small $\epsilon>0$, then the corresponding $\alpha$ can be decomposed as

$$
\alpha(x, y)=\frac{1}{2}(\psi(x)+\psi(y)) \alpha^{0}\left(h^{-1}(x-y)\right)+\sigma(x, y)
$$

with $\mathcal{E}^{\mathrm{GL}}(\psi) \leq E^{\mathrm{GL}}+\epsilon+$ const $h^{\frac{1}{3}}, \alpha^{0}$ defined in (1.11), and

$$
\int_{\mathcal{C} \times \mathbb{R}}|\sigma(x, y)|^{2} d x d y \leq \text { const } h^{3+\frac{1}{3}}
$$

1.5. The coefficients in the GL functional. In order to give explicit expressions for the coefficients in the GL functional we introduce the functions

$$
g_{0}(z)=\frac{\tanh (z / 2)}{z}, \quad g_{1}(z)=\frac{e^{2 z}-2 z e^{z}-1}{z^{2}\left(1+e^{z}\right)^{2}}, \quad g_{2}(z)=\frac{2 e^{z}\left(e^{z}-1\right)}{z\left(e^{z}+1\right)^{3}} .
$$

Setting, as usual, $\beta_{c}=T_{c}^{-1}$ we define

$$
c=\frac{2 \int_{\mathbb{R}}\left[g_{0}\left(\beta_{c}\left(q^{2}-\mu\right)\right)-\beta_{c}\left(q^{2}-\mu\right) g_{1}\left(\beta_{c}\left(q^{2}-\mu\right)\right)\right] d q}{\beta_{c} \int_{\mathbb{R}} \frac{g_{1}\left(\beta_{c}\left(q^{2}-\mu\right)\right)}{q^{2}-\mu} d q} .
$$

The three coefficients of the G-L functional turn out to be as follows,

$$
\begin{gathered}
b_{1}=c D \frac{\beta_{c}^{2}}{16} \int_{\mathbb{R}}\left(g_{1}\left(\beta_{c}\left(q^{2}-\mu\right)\right)+2 \beta_{c} q^{2} g_{2}\left(\beta_{c}\left(q^{2}-\mu\right)\right)\right) \frac{d q}{2 \pi}, \\
b_{2}=c D \frac{\beta_{c}^{2}}{4} \int_{\mathbb{R}} g_{1}\left(\beta_{c}\left(q^{2}-\mu\right)\right) \frac{d q}{2 \pi}
\end{gathered}
$$

and

$$
b_{3}=(c D)^{2} \frac{\beta_{c}^{2}}{16} \int_{\mathbb{R}} \frac{g_{1}\left(\beta_{c}\left(q^{2}-\mu\right)\right)}{q^{2}-\mu} \frac{d q}{2 \pi} .
$$

We shall now discuss the signs of these coefficients. First note that $g_{0}(z)-$ $z g_{1}(z)=\left(z g_{0}(z)\right)^{\prime}>0$ and $g_{1}(z) / z>0$, which implies that $c>0$. Using $g_{1}(z) / z>$ 0 again, we see that $b_{3}>0$. In contrast, the coefficient $b_{2}$ may have either sign, depending on the value of $\beta_{c} \mu$ (which depends on $a$ and $\mu$ ). The coefficient $b_{1}$ is 
again positive, as the following computation shows: using the fact that $g_{2}(z)=$ $g_{1}^{\prime}(z)+(2 / z) g_{1}(z)$ we find

$$
\begin{aligned}
b_{1} & =c D \frac{\beta_{c}^{2}}{16} \int_{\mathbb{R}}\left(g_{1}\left(\beta_{c}\left(q^{2}-\mu\right)\right)+2 \beta_{c} q^{2}\left(g_{1}^{\prime}\left(\beta_{c}\left(q^{2}-\mu\right)\right)+\frac{2 g_{1}\left(\beta_{c}\left(q^{2}-\mu\right)\right)}{\beta_{c}\left(q^{2}-\mu\right)}\right)\right) \frac{d q}{2 \pi} \\
& =c D \frac{\beta_{c}^{2}}{16} \int_{\mathbb{R}}\left(g_{1}\left(\beta_{c}\left(q^{2}-\mu\right)\right)+q \frac{d}{d q}\left(g_{1}\left(\beta_{c}\left(q^{2}-\mu\right)\right)\right)+4 q^{2} \frac{g_{1}\left(\beta_{c}\left(q^{2}-\mu\right)\right)}{q^{2}-\mu}\right) \frac{d q}{2 \pi} \\
& =c D \frac{\beta_{c}^{2}}{4} \int_{\mathbb{R}} q^{2} \frac{g_{1}\left(\beta_{c}\left(q^{2}-\mu\right)\right)}{q^{2}-\mu} \frac{d q}{2 \pi} .
\end{aligned}
$$

The claimed positivity is now again a consequence of $g_{1}(z) / z>0$.

\section{Sketch of the PROOF}

In the following we will consider temperatures $T=T_{c}\left(1-D h^{2}\right)$. It is not difficult to see that the solution $\Delta_{0}$ of the BCS gap equation (1.7) is of order $\Delta_{0}=O(h)$.

It is useful to rewrite the BCS functional in a more convenient way. Define $\Delta$ to be the multiplication operator

$$
\Delta=\Delta(x)=-\psi(x) \Delta_{0},
$$

where $\Delta_{0}$ is the solution of the BCS equation (1.7) for temperature $T$, and $\psi$ a periodic function in $H_{\mathrm{loc}}^{2}(\mathbb{R})$. Define further

$$
H_{\Delta}=\left(\begin{array}{cc}
-h^{2} \nabla^{2}-\mu+h^{2} W(x) & \Delta \\
\Delta & h^{2} \nabla^{2}+\mu-h^{2} W(x)
\end{array}\right) .
$$

Formally, we can write the BCS functional as

$$
\begin{aligned}
\mathcal{F}^{\mathrm{BCS}}(\Gamma)=-\operatorname{Tr}( & \left.-h^{2} \nabla^{2}-\mu+h^{2} W\right)+\frac{1}{2} \operatorname{Tr} H_{\Delta} \Gamma-T S(\Gamma) \\
& +\frac{1}{4 h a} \Delta_{0}^{2} \int_{\mathcal{C}}|\psi(x)|^{2} d x-h a \int_{\mathcal{C}}\left|\frac{\Delta_{0} \psi(x)}{2 h a}-\alpha(x, x)\right|^{2} d x .
\end{aligned}
$$

The first two terms on the right are infinite, of course, only their sum is well-defined. For an upper bound, we can drop the very last term. The terms on the first line are minimized for $\Gamma_{\Delta}=1 /\left(1+e^{\frac{1}{T} H_{\Delta}}\right)$, which we choose as a trial state. Then

$$
\begin{aligned}
F^{\mathrm{BCS}}(T, \mu) & \leq \mathcal{F}^{\mathrm{BCS}}\left(\Gamma_{\Delta}\right)-\mathcal{F}^{\mathrm{BCS}}\left(\Gamma_{0}\right) \\
& \leq-\frac{T}{2} \operatorname{Tr}\left[\ln \left(1+e^{-\frac{1}{T} H_{\Delta}}\right)-\ln \left(1+e^{-\frac{1}{T} H_{0}}\right)\right]+\frac{1}{4 h a} \Delta_{0}^{2} \int_{\mathcal{C}}|\psi(x)|^{2} d x .
\end{aligned}
$$

To complete the upper bound, we have to evaluate $\operatorname{Tr}\left[\ln \left(1+e^{-H_{\Delta} / T}\right)-\ln (1+\right.$ $\left.e^{-H_{0} / T}\right)$. This is done via a contour integral representation and semiclassical types of estimates.

The lower bound is divided into several steps. We first aim at an a priori bound on $\alpha$ for a general state $\Gamma$, which has lower energy than the translation-invariant state. With

$$
H_{\Delta_{0}}^{0}=\left(\begin{array}{cc}
-h^{2} \nabla^{2}-\mu & \Delta_{0} \\
\Delta_{0} & -h^{2} \nabla^{2}+\mu
\end{array}\right)
$$


we can rewrite the BCS functional in the form

$$
\begin{aligned}
\mathcal{F}^{\mathrm{BCS}}(\Gamma)=-\operatorname{Tr}\left(-h^{2} \nabla^{2}-\mu+h^{2} W\right) & +\frac{1}{2} \operatorname{tr} H_{\Delta_{0}}^{0} \Gamma-T S(\Gamma) \\
& +h^{2} \operatorname{Tr} W \gamma+\frac{1}{4 h a} \Delta_{0}^{2}-h a \int_{\mathcal{C}}\left|\frac{\Delta_{0}}{2 h a}-\alpha(x, x)\right|^{2} d x .
\end{aligned}
$$

From the BCS equation and the definition of $\alpha^{0}$ in (1.11) we conclude that

$$
\alpha^{0}(0)=\frac{1}{2 \pi h} \int_{\mathbb{R}} \frac{\Delta_{0}}{2 K_{T}^{0}(p)} d p=\frac{\Delta_{0}}{2 a h},
$$

and hence

$\mathcal{F}^{\mathrm{BCS}}(\Gamma)-\mathcal{F}^{\mathrm{BCS}}\left(\Gamma^{0}\right) \geq \frac{T}{2} \mathcal{H}\left(\Gamma, \Gamma^{0}\right)+h^{2} \operatorname{Tr} W\left(\gamma-\gamma^{0}\right)-a h \int_{\mathcal{C}}\left|\alpha(x, x)-\alpha^{0}(0)\right|^{2} d x$,

where $\mathcal{H}$ denotes the relative entropy

$$
\begin{aligned}
\mathcal{H}\left(\Gamma, \Gamma^{0}\right) & =\frac{2}{T}\left(\frac{1}{2} \operatorname{tr} H_{\Delta_{0}}^{0} \Gamma-T S(\Gamma)+\frac{1}{2} \operatorname{tr} H_{\Delta_{0}}^{0} \Gamma^{0}-T S\left(\Gamma^{0}\right)\right) \\
& =\operatorname{Tr}\left[\Gamma\left(\ln \Gamma-\ln \Gamma^{0}\right)+(1-\Gamma)\left(\ln (1-\Gamma)-\ln \left(1-\Gamma^{0}\right)\right)\right] .
\end{aligned}
$$

Note that the left side of (2.6) is necessarily non-positive for a minimizing state $\Gamma$.

One of the essential steps in our proof, which is used on several occasions, is Lemma 1. This Lemma presents a lower bound on the relative entropy of the form

$$
\begin{aligned}
\mathcal{H}\left(\Gamma, \Gamma^{0}\right) \geq & \operatorname{Tr}\left[H^{0}\left(\Gamma-\Gamma^{0}\right)^{2}\right] \\
& +\frac{1}{3} \frac{\left(\operatorname{Tr} \Gamma(1-\Gamma)-\operatorname{Tr} \Gamma_{0}\left(1-\Gamma^{0}\right)\right)^{2}}{\left|\operatorname{Tr} \Gamma(1-\Gamma)-\operatorname{Tr} \Gamma^{0}\left(1-\Gamma^{0}\right)\right|+\operatorname{Tr} \Gamma^{0}\left(1-\Gamma^{0}\right)},
\end{aligned}
$$

where $H^{0}=\left(1-2 \Gamma_{0}\right)^{-1} \ln \left(\left(1-\Gamma^{0}\right) / \Gamma^{0}\right)$. In our case here, it equals $K_{T}^{0}(-i h \nabla) / T$, with $K_{T}^{0}$ defined in (1.8). From (2.6) we deduce that for a minimizer $\Gamma$

$$
\begin{aligned}
0 \geq & \mathcal{F}^{\mathrm{BCS}}(\Gamma)-\mathcal{F}^{\mathrm{BCS}}\left(\Gamma^{0}\right) \geq \operatorname{Tr} K_{T}^{0}\left(\gamma-\gamma^{0}\right)^{2}+h^{2} \operatorname{Tr} W\left(\gamma-\gamma^{0}\right) \\
& +\int_{\mathcal{C}}\left\langle\alpha(\cdot, y)-\alpha^{0}\left(\frac{\cdot-y}{h}\right)\left|K_{T}^{0}(-i h \nabla)-a \delta\left(\frac{-y}{h}\right)\right| \alpha(\cdot, y)-\alpha^{0}\left(\frac{-y}{h}\right)\right\rangle d y \\
+ & \frac{1}{3} \frac{T\left(\operatorname{Tr}\left[\gamma(1-\gamma)-\gamma^{0}\left(1-\gamma^{0}\right)-|\alpha|^{2}+\left|\alpha^{0}\right|^{2}\right]\right)^{2}}{\left|\operatorname{Tr}\left[\gamma(1-\gamma)-\gamma^{0}\left(1-\gamma^{0}\right)-|\alpha|^{2}+\left|\alpha^{0}\right|^{2}\right]\right|+\operatorname{Tr}\left[\gamma^{0}\left(1-\gamma^{0}\right)-\left|\alpha^{0}\right|^{2}\right]},
\end{aligned}
$$

where $\langle\cdot \mid \cdot\rangle$ denotes the inner product in $L^{2}(\mathbb{R})$. Observe that the term in the second line is a convenient way to write $\operatorname{Tr} K_{T}^{0}\left(\alpha-\alpha^{0}\right)^{*}\left(\alpha-\alpha^{0}\right)-a h \int_{\mathcal{C}}\left|\alpha(x, x)-\alpha^{0}(0)\right|^{2} d x$. From the first line on the right side and the Schwarz inequality together with the fact that $K_{T}^{0}-a \delta \geq 0$, we obtain first that $\operatorname{Tr} K_{T}^{0}\left(\gamma-\gamma^{0}\right)^{2} \leq O\left(h^{3}\right)$. Together with the last line this further gives the a priori bound $\|\alpha\|_{2}^{2} \leq O(h)$.

Next, we use that $K_{T}^{0}-a \delta$ has $\alpha^{0}$ as unique zero energy ground state, with a gap of order one above zero, and we can further conclude from (2.9) that $\alpha$ is necessarily of the form

$$
\alpha(x, y)=\frac{1}{2}(\psi(x)+\psi(y)) \alpha^{0}((x-y) / h)+\beta(x, y),
$$

with $\|\beta\|_{2}^{2} \leq O\left(h^{3}\right)$. This information about the decomposition of $\alpha$ then allows us to deduce, again by means of a lower bound of the type (2.8), that the difference 
$\mathcal{F}^{\mathrm{BCS}}(\Gamma)-\mathcal{F}^{\mathrm{BCS}}\left(\Gamma_{\Delta}\right)$ is very small compared to $h^{3}$. This reduces the problem to the computation we already did in the upper bound.

\section{Semiclassics}

One of the key ingredients in both the proof of the upper and the lower bound are detailed semiclassical asymptotics for operators of the form

$$
H_{\Delta}=\left(\begin{array}{cc}
-h^{2} \nabla^{2} \frac{-\mu+h^{2} W(x)}{\Delta(x)} & \Delta(x) \\
& h^{2} \nabla^{2}+\mu-h^{2} W(x)
\end{array}\right) .
$$

Here $\Delta(x)=-h \psi(x)$ with a periodic function $\psi$, which is of order one as $h \rightarrow$ 0 (but might nevertheless depend on $h$ ). We are interested in the regime $h \rightarrow$ 0 . In contrast to traditional semiclassical results [12, 16, we work under minimal smoothness assumptions on $\psi$ and $W$. To be precise, we assume Assumption 1 for $W$ and that $\psi$ is a periodic function in $H_{\mathrm{loc}}^{2}(\mathbb{R})$.

Our first result concerns the free energy.

THEOREM 2. Let

$$
f(z)=-\ln \left(1+e^{-z}\right)
$$

and define

$$
\begin{gathered}
g_{0}(z)=\frac{f^{\prime}(-z)-f^{\prime}(z)}{z}=\frac{\tanh \left(\frac{1}{2} z\right)}{z} \\
g_{1}(z)=-g_{0}^{\prime}(z)=\frac{f^{\prime}(-z)-f^{\prime}(z)}{z^{2}}+\frac{f^{\prime \prime}(-z)+f^{\prime \prime}(z)}{z}=\frac{e^{2 z}-2 z e^{z}-1}{z^{2}\left(1+e^{z}\right)^{2}}
\end{gathered}
$$

and

$$
g_{2}(z)=g_{1}^{\prime}(z)+\frac{2}{z} g_{1}(z)=\frac{f^{\prime \prime \prime}(z)-f^{\prime \prime \prime}(-z)}{z}=\frac{2 e^{z}\left(e^{z}-1\right)}{z\left(e^{z}+1\right)^{3}} .
$$

Then, for any $\beta>0$,

$$
\frac{h}{\beta} \operatorname{Tr}\left[f\left(\beta H_{\Delta}\right)-f\left(\beta H_{0}\right)\right]=h^{2} E_{1}+h^{4} E_{2}+O\left(h^{6}\right)\left(\|\psi\|_{H^{1}(\mathcal{C})}^{6}+\|\psi\|_{H^{2}(\mathcal{C})}^{2}\right),
$$

where

$$
E_{1}=-\frac{\beta}{2}\|\psi\|_{2}^{2} \int_{\mathbb{R}} g_{0}\left(\beta\left(q^{2}-\mu\right)\right) \frac{d q}{2 \pi}
$$

and

$$
\begin{aligned}
E_{2}= & \frac{\beta^{2}}{8}\left\|\psi^{\prime}\right\|_{2}^{2} \int_{\mathbb{R}}\left(g_{1}\left(\beta\left(q^{2}-\mu\right)\right)+2 \beta q^{2} g_{2}\left(\beta\left(q^{2}-\mu\right)\right)\right) \frac{d q}{2 \pi} \\
& +\frac{\beta^{2}}{2}\langle\psi|W| \psi\rangle \int_{\mathbb{R}} g_{1}\left(\beta\left(q^{2}-\mu\right)\right) \frac{d q}{2 \pi} \\
& +\frac{\beta^{2}}{8}\|\psi\|_{4}^{4} \int_{\mathbb{R}} \frac{g_{1}\left(\beta\left(q^{2}-\mu\right)\right)}{q^{2}-\mu} \frac{d q}{2 \pi} .
\end{aligned}
$$

More precisely, we claim that the diagonal entries of the $2 \times 2$ matrix-valued operator $f\left(\beta H_{\Delta}\right)-f\left(\beta H_{0}\right)$ are locally trace class and that the sum of their traces per unit volume is given by (3.6). We sketch the proof of Theorem 2 in Subsection 6.2 below and refer to 7 for some technicalities.

Our second semiclassical result concerns the behavior of $\left(1+\exp \left(\beta H_{\Delta}\right)\right)^{-1}$ in the limit $h \rightarrow 0$. More precisely, we are interested in $\left[\left(1+\exp \left(\beta H_{\Delta}\right)\right)^{-1}\right]_{12}$, where 
$[\cdot]_{12}$ denotes the upper off-diagonal entry of an operator-valued $2 \times 2$ matrix. For this purpose, we define the $H^{1}$ norm of a periodic operator $\eta$ by

$$
\|\eta\|_{H^{1}}^{2}=\operatorname{Tr}\left[\eta^{*}\left(1-h^{2} \nabla^{2}\right) \eta\right] .
$$

In Subsection 6.3 we shall prove

THEOREM 3. Let

$$
\rho(z)=\left(1+e^{z}\right)^{-1}
$$

and let $g_{0}$ be as in (3.3). Then

$$
\left[\rho\left(\beta H_{\Delta}\right)\right]_{12}=\frac{\beta h}{4}\left(\psi(x) g_{0}\left(\beta\left(-h^{2} \nabla^{2}-\mu\right)\right)+g_{0}\left(\beta\left(-h^{2} \nabla^{2}-\mu\right)\right) \psi(x)\right)+\eta_{1}+\eta_{2}
$$

where

and

$$
\left\|\eta_{1}\right\|_{H^{1}}^{2} \leq C h^{5}\|\psi\|_{H^{2}(\mathcal{C})}^{2}
$$

$$
\left\|\eta_{2}\right\|_{H^{1}}^{2} \leq C h^{5}\left(\|\psi\|_{H^{1}(\mathcal{C})}^{2}+\|\psi\|_{H^{1}(\mathcal{C})}^{6}\right) \text {. }
$$

\section{UPPER BOUND}

We assume that $T=T_{c}\left(1-D h^{2}\right)$ with a fixed $D>0$ and denote by $\Delta_{0}$ the solution of the BCS gap equation (1.7). In the following we write, as usual, $\beta=$ $T^{-1}=\beta_{c}\left(1-D h^{2}\right)^{-1}$ with $\beta_{c}=T_{c}^{-1}$. It is well known that the Ginzburg-Landau functional has a minimizer $\psi$, which is a periodic $H_{\mathrm{loc}}^{2}(\mathbb{R})$ function. We put

$$
\Delta(x)=-\Delta_{0} \psi(x),
$$

and define $H_{\Delta}$ by (2.1).

To obtain an upper bound for the energy we use the trial state

$$
\Gamma_{\Delta}=\left(1+e^{\beta H_{\Delta}}\right)^{-1} .
$$

Denoting its off-diagonal element by $\alpha_{\Delta}=\left[\Gamma_{\Delta}\right]_{12}$, we have the upper bound

$$
\begin{aligned}
\mathcal{F}^{\mathrm{BCS}}\left(\Gamma_{\Delta}\right)-\mathcal{F}^{\mathrm{BCS}}\left(\Gamma_{0}\right)= & -\frac{1}{2 \beta} \operatorname{Tr}\left[\ln \left(1+e^{-\beta H_{\Delta}}\right)-\ln \left(1+e^{-\beta H_{0}}\right)\right] \\
& +\frac{\Delta_{0}^{2}}{4 h a}\|\psi\|_{2}^{2}-h a \int_{C}\left|\frac{\Delta_{0} \psi(x)}{2 h a}-\alpha_{\Delta}(x, x)\right|^{2} d x \\
\leq & -\frac{1}{2 \beta} \operatorname{Tr}\left[\ln \left(1+e^{-\beta H_{\Delta}}\right)-\ln \left(1+e^{-\beta H_{0}}\right)\right]+\frac{\Delta_{0}^{2}}{4 h a}\|\psi\|_{2}^{2} .
\end{aligned}
$$

The first term on the right side was evaluated in Theorem2 Applying this theorem with $\psi$ replaced by $\left(\Delta_{0} / h\right) \psi$ we obtain that

$$
\begin{aligned}
\mathcal{F}^{\mathrm{BCS}}\left(\Gamma_{\Delta}\right)-\mathcal{F}^{\mathrm{BCS}}\left(\Gamma_{0}\right) \\
\leq-\frac{h \beta}{4} \frac{\Delta_{0}^{2}}{h^{2}}\|\psi\|_{2}^{2} \int_{\mathbb{R}} g_{0}\left(\beta\left(q^{2}-\mu\right)\right) \frac{d q}{2 \pi} \\
+\frac{h^{3}}{2}\left[\frac{\beta^{2}}{8} \frac{\Delta_{0}^{2}}{h^{2}}\left\|\psi^{\prime}\right\|_{2}^{2} \int_{\mathbb{R}}\left(g_{1}\left(\beta\left(q^{2}-\mu\right)\right)+2 \beta q^{2} g_{2}\left(\beta\left(q^{2}-\mu\right)\right)\right) \frac{d q}{2 \pi}\right. \\
\left.\quad+\frac{\beta^{2}}{2} \frac{\Delta_{0}^{2}}{h^{2}}\langle\psi|W| \psi\rangle \int_{\mathbb{R}} g_{1}\left(\beta\left(q^{2}-\mu\right)\right) \frac{d q}{2 \pi}+\frac{\beta^{2}}{8} \frac{\Delta_{0}^{4}}{h^{4}}\|\psi\|_{4}^{4} \int_{\mathbb{R}} \frac{g_{1}\left(\beta\left(q^{2}-\mu\right)\right)}{q^{2}-\mu} \frac{d q}{2 \pi}\right] \\
+\frac{\Delta_{0}^{2}}{4 h a}\|\psi\|_{2}^{2}+O\left(h^{5}\right) .
\end{aligned}
$$


In the estimate of the remainder we used that $\psi$ is $H^{2}$ and that $\Delta_{0} \leq C h$.

Next, we use that by definition (1.7) of $\Delta_{0}$ the first and the last term on the right side of (4.2) cancel to leading order and that one has

$$
\begin{aligned}
- & \frac{h \beta}{4} \frac{\Delta_{0}^{2}}{h^{2}}\|\psi\|_{2}^{2} \int_{\mathbb{R}} g_{0}\left(\beta\left(q^{2}-\mu\right)\right) \frac{d q}{2 \pi}+\frac{\Delta_{0}^{2}}{4 h a}\|\psi\|_{2}^{2} \\
= & \frac{h \beta}{4} \frac{\Delta_{0}^{2}}{h^{2}}\|\psi\|_{2}^{2} \int_{\mathbb{R}}\left(g_{0}\left(\beta \sqrt{\left(q^{2}-\mu\right)^{2}+\Delta_{0}^{2}}\right)-g_{0}\left(\beta\left(q^{2}-\mu\right)\right)\right) \frac{d q}{2 \pi} \\
= & -\frac{h^{3} \beta^{2}}{8} \frac{\Delta_{0}^{4}}{h^{4}}\|\psi\|_{2}^{2} \int_{\mathbb{R}} \frac{g_{1}\left(\beta\left(q^{2}-\mu\right)\right)}{q^{2}-\mu} \frac{d q}{2 \pi}+O\left(h^{5}\right) .
\end{aligned}
$$

We conclude that

$$
\begin{aligned}
\mathcal{F}^{\mathrm{BCS}}\left(\Gamma_{\Delta}\right)-\mathcal{F}^{\mathrm{BCS}}\left(\Gamma_{0}\right) \\
\leq \frac{h^{3}}{2}\left[\frac{\beta^{2}}{8} \frac{\Delta_{0}^{2}}{h^{2}}\left\|\psi^{\prime}\right\|_{2}^{2} \int_{\mathbb{R}}\left(g_{1}\left(\beta\left(q^{2}-\mu\right)\right)+2 \beta q^{2} g_{2}\left(\beta\left(q^{2}-\mu\right)\right)\right) \frac{d q}{2 \pi}\right. \\
\quad+\frac{\beta^{2}}{2} \frac{\Delta_{0}^{2}}{h^{2}}\langle\psi|W| \psi\rangle \int_{\mathbb{R}} g_{1}\left(\beta\left(q^{2}-\mu\right)\right) \frac{d q}{2 \pi}+\frac{\beta^{2}}{8} \frac{\Delta_{0}^{4}}{h^{4}}\|\psi\|_{4}^{4} \int_{\mathbb{R}} \frac{g_{1}\left(\beta\left(q^{2}-\mu\right)\right)}{q^{2}-\mu} \frac{d q}{2 \pi} \\
\left.\quad-\frac{\beta^{2}}{4} \frac{\Delta_{0}^{4}}{h^{4}}\|\psi\|_{2}^{2} \int_{\mathbb{R}} \frac{g_{1}\left(\beta\left(q^{2}-\mu\right)\right)}{q^{2}-\mu} \frac{d q}{2 \pi}\right]+O\left(h^{5}\right) .
\end{aligned}
$$

Up to an error of the order $O\left(h^{5}\right)$ we can replace $\beta=\beta_{c}\left(1-D h^{2}\right)^{-1}$ by $\beta_{c}$ on the right side. Our last task is then to compute the asymptotics of $\Delta_{0} / h$. To do so, we rewrite the BCS gap equation (1.7) as

$$
\beta_{c} \int_{\mathbb{R}} g_{0}\left(\beta_{c}\left(q^{2}-\mu\right)\right) \frac{d q}{2 \pi}=\frac{1}{a}=\beta \int_{\mathbb{R}} g_{0}\left(\beta \sqrt{\left(q^{2}-\mu\right)^{2}+\Delta_{0}^{2}}\right) \frac{d q}{2 \pi} .
$$

A simple computation shows that

$$
\Delta_{0}^{2}=D h^{2} \frac{\int_{\mathbb{R}}\left[g_{0}\left(\beta_{c}\left(q^{2}-\mu\right)\right)-\beta_{c}\left(q^{2}-\mu\right) g_{1}\left(\beta_{c}\left(q^{2}-\mu\right)\right)\right] d q}{\beta_{c} \int_{\mathbb{R}} \frac{g_{1}\left(\beta_{c}\left(q^{2}-\mu\right)\right)}{2\left(q^{2}-\mu\right)} d q}\left(1+O\left(h^{2}\right)\right) .
$$

Inserting this into (4.3) and using the fact that $\mathcal{E}(\psi)=E^{\mathrm{GL}}$ we arrive at the upper bound claimed in Theorem 1 .

\section{LOWER BOUnd}

5.1. The relative entropy. As a preliminary to our proof of the lower bound, we present a general estimate for the relative entropy. In this subsection $H^{0}$ and $0 \leq \Gamma \leq 1$ are arbitrary self-adjoint operators in a Hilbert space, not necessarily coming from BCS theory. Let $\Gamma^{0}:=\left(1+\exp \left(\beta H^{0}\right)\right)^{-1}$. It is well-known that

$$
\mathcal{H}\left(\Gamma, \Gamma^{0}\right)=\operatorname{Tr}\left(\beta H^{0} \Gamma+\Gamma \ln \Gamma+(1-\Gamma) \ln (1-\Gamma)+\ln \left(1+\exp \left(-\beta H_{0}\right)\right)\right)
$$

is non-negative and equals to zero if and only if $\Gamma=\Gamma^{0}$. Solving this equation for $H^{0}$, i.e., $H^{0}=\beta^{-1}\left(\ln \left(1-\Gamma^{0}\right)-\ln \Gamma^{0}\right)$, we can rewrite $\mathcal{H}\left(\Gamma, \Gamma^{0}\right)$ as a relative entropy,

$$
\mathcal{H}\left(\Gamma, \Gamma^{0}\right)=\operatorname{Tr}\left[\Gamma\left(\ln \Gamma-\ln \Gamma^{0}\right)+(1-\Gamma)\left(\ln (1-\Gamma)-\ln \left(1-\Gamma^{0}\right)\right)\right] .
$$

The following lemma quantifies the positivity of $\mathcal{H}$ and improves an earlier result from 5 . 
Lemma 1. For any $0 \leq \Gamma \leq 1$ and any $\Gamma_{0}$ of the form $\Gamma^{0}=\left(1+e^{\beta H^{0}}\right)^{-1}$,

$$
\begin{aligned}
\mathcal{H}\left(\Gamma, \Gamma^{0}\right) \geq & \operatorname{Tr}\left[\frac{\beta H^{0}}{\tanh \left(\beta H^{0} / 2\right)}\left(\Gamma-\Gamma^{0}\right)^{2}\right] \\
& +\frac{1}{3} \frac{\left(\operatorname{Tr} \Gamma(1-\Gamma)-\operatorname{Tr} \Gamma^{0}\left(1-\Gamma^{0}\right)\right)^{2}}{\operatorname{Tr} \Gamma(1-\Gamma)-\operatorname{Tr} \Gamma^{0}\left(1-\Gamma^{0}\right) \mid+\operatorname{Tr} \Gamma^{0}\left(1-\Gamma^{0}\right)} .
\end{aligned}
$$

Proof. It is tedious, but elementary, to show that for real numbers $0<x, y<1$,

$$
x \ln \frac{x}{y}+(1-x) \ln \frac{1-x}{1-y} \geq \frac{\ln \frac{1-y}{y}}{1-2 y}(x-y)^{2}+\frac{1}{3} \frac{(x(1-x)-y(1-y))^{2}}{|x(1-x)-y(1-y)|+y(1-y)} .
$$

Using joint convexity we see that

$$
\begin{aligned}
\frac{(x(1-x)-y(1-y))^{2}}{|x(1-x)-y(1-y)|+y(1-y)} & \\
= & 4 \sup _{0<b<1}\left[b(1-b)|x(1-x)-y(1-y)|-b^{2} y(1-y)\right] .
\end{aligned}
$$

Let us replace on the right side the modulus $|a|$ by $\max \{a,-a\}$, and then use Klein's inequality [15, Section 2.1.4] for either of the expressions. This implies the result.

5.2. A priori estimates on $\alpha$. We begin by briefly reviewing some facts about the translation-invariant case $W \equiv 0$; see also Subsection 1.2.1. Recall that $\Gamma^{0}$ denotes the minimizer of $\mathcal{F}$ in the translation-invariant case. It can be written as $\Gamma^{0}=\left(1+e^{\beta H_{\Delta_{0}}^{0}}\right)^{-1}$ with

$$
H_{\Delta_{0}}^{0}=\left(\begin{array}{cc}
-h^{2} \nabla^{2}-\mu & -\Delta_{0} \\
-\Delta_{0} & h^{2} \nabla^{2}+\mu
\end{array}\right) .
$$

Here $\Delta_{0}$ is the solution of the BCS gap-equation (1.7) and $\beta^{-1}=T=T_{c}\left(1-D h^{2}\right)$. Notice the distinction between $\Gamma^{0}$ and $\Gamma_{0}$ which was defined in (1.13). The latter one, $\Gamma_{0}$, contains the external potential $W$ and has no off-diagonal term.

Recall also that we denote the kernel of the off-diagonal entry $\alpha^{0}=\left[\Gamma^{0}\right]_{12}$ by $\alpha^{0}((x-y) / h)$, which is explicitly given in (1.11). From this explicit representation and the fact that $\Delta_{0} \leq C h$ we conclude, in particular, that

$$
\left\|\alpha^{0}\right\|_{2}^{2}=\int_{0}^{1} d y \int_{\mathbb{R}} d x\left|\alpha^{0}((x-y) / h)\right|^{2}=h \int_{\mathbb{R}}\left|\alpha^{0}(x)\right|^{2} d x \leq C h .
$$

Moreover, the BCS gap-equation (1.7) is equivalent to

$$
\left(K_{T}^{0}(-i h \nabla)-a h \delta(x)\right) \alpha^{0}(x / h)=0 .
$$

This implies, in particular, that

$$
\operatorname{Tr} K_{T}^{0}(-i h \nabla) \alpha^{0} \overline{\alpha^{0}}-a h\left|\alpha^{0}(0)\right|^{2}=0 .
$$

Now we turn to the case of general $W$. Our goal in this subsection is to prove that the $\alpha$ of any low-energy state satisfies bounds similar to (5.2) and (5.4).

Proposition 1. Any admissible $\Gamma$ with $\mathcal{F}^{\mathrm{BCS}}(\Gamma) \leq \mathcal{F}^{\mathrm{BCS}}\left(\Gamma^{0}\right)$ satisfies

$$
\|\alpha\|_{2}^{2}=\int_{0}^{1} d x \int_{\mathbb{R}} d y|\alpha(x, y)|^{2} \leq C h
$$


and

$$
0 \leq \operatorname{Tr} K_{T}^{0}(-i h \nabla) \alpha \bar{\alpha}-a h \int_{\mathcal{C}}|\alpha(x, x)|^{2} d x \leq C h^{3},
$$

where $\alpha=[\Gamma]_{12}$.

Proof. We divide the proof into two steps.

Step 1. Our starting point is the representation

$$
\mathcal{F}^{\mathrm{BCS}}(\Gamma)-\mathcal{F}^{\mathrm{BCS}}\left(\Gamma^{0}\right)=\frac{1}{2 \beta} \mathcal{H}\left(\Gamma, \Gamma^{0}\right)+h^{2} \operatorname{Tr} \gamma W-a h \int_{\mathcal{C}}\left|\alpha(x, x)-\alpha^{0}(0)\right|^{2} d x
$$

for any admissible $\Gamma$, with the relative entropy $\mathcal{H}\left(\Gamma, \Gamma^{0}\right)$ defined in (5.1). We note that $\Gamma^{0}$ is of the form $\left(1+e^{\beta H^{0}}\right)^{-1}$ with $H^{0}=H_{\Delta_{0}}^{0}$. We use Lemma 1 to bound $\mathcal{H}\left(\Gamma, \Gamma^{0}\right)$ from below. Since $x \mapsto x / \tanh x$ is even, we can replace $H_{\Delta_{0}}^{0}$ by its absolute value $E(-i h \nabla)=\sqrt{\left(-h^{2} \nabla^{2}-\mu\right)^{2}+\Delta_{0}^{2}}$, and thus

$$
\begin{aligned}
& \operatorname{Tr}\left[\frac{H_{\Delta_{0}}^{0}}{\tanh \frac{\beta}{2} H_{\Delta_{0}}^{0}}\left(\Gamma-\Gamma_{0}\right)^{2}\right]=\operatorname{Tr}\left[K_{T}^{0}(-i h \nabla)\left(\Gamma-\Gamma_{0}\right)^{2}\right] \\
& \quad=2 \operatorname{Tr} K_{T}^{0}(-i h \nabla)\left(\gamma-\gamma^{0}\right)^{2}+2 \operatorname{Tr} K_{T}^{0}(-i h \nabla)\left(\alpha-\alpha^{0}\right)\left(\overline{\alpha-\alpha^{0}}\right)
\end{aligned}
$$

with

$$
K_{T}^{0}(h p)=\frac{E(h p)}{\tanh \frac{\beta E(h p)}{2}}
$$

from (1.8). With the aid of Lemma 1 and the assumption $\mathcal{F}^{\mathrm{BCS}}(\Gamma) \leq \mathcal{F}^{\mathrm{BCS}}\left(\Gamma^{0}\right)$ we obtain from (5.7) the basic inequality

$$
\begin{aligned}
0 \geq & \operatorname{Tr} K_{T}^{0}(-i h \nabla)\left(\gamma-\gamma^{0}\right)^{2}+h^{2} \operatorname{Tr} \gamma W \\
& +\operatorname{Tr} K_{T}^{0}(-i h \nabla)\left(\alpha-\alpha^{0}\right)\left(\overline{\alpha-\alpha^{0}}\right)-a h \int_{\mathcal{C}}\left|\alpha(x, x)-\alpha^{0}(0)\right|^{2} d x \\
& +\frac{T}{3} \frac{\left(\operatorname{Tr}\left[\gamma(1-\gamma)-\gamma^{0}\left(1-\gamma^{0}\right)-\alpha \bar{\alpha}+\alpha^{0} \overline{\alpha^{0}}\right]\right)^{2}}{\operatorname{Tr}\left[\gamma(1-\gamma)-\gamma^{0}\left(1-\gamma^{0}\right)-\alpha \bar{\alpha}+\alpha^{0} \overline{\alpha^{0}}\right] \mid+\operatorname{Tr}\left[\gamma^{0}\left(1-\gamma^{0}\right)-\alpha^{0} \overline{\alpha^{0}}\right]} .
\end{aligned}
$$

In the following step we shall derive the claimed a priori estimates on $\alpha$ from this inequality.

Step 2. We begin by discussing the first line on the right side of (5.8). Using the fact that $\operatorname{Tr} W \gamma^{0}=0$ (since $W$ has mean value zero) and the Schwarz inequality we obtain the lower bound

$$
\begin{aligned}
\operatorname{Tr} & K_{T}^{0}(-i h \nabla)\left(\gamma-\gamma^{0}\right)^{2}+h^{2} \operatorname{Tr} W \gamma \\
\quad & =\frac{1}{2} \operatorname{Tr} K_{T}^{0}(-i h \nabla)\left(\gamma-\gamma^{0}\right)^{2}+\frac{1}{2} \operatorname{Tr} K_{T}^{0}\left(\gamma-\gamma^{0}\right)^{2}+h^{2} \operatorname{Tr} W\left(\gamma-\gamma^{0}\right) \\
\quad \geq & \frac{1}{2} \operatorname{Tr} K_{T}^{0}(-i h \nabla)\left(\gamma-\gamma^{0}\right)^{2}-h^{4} \frac{1}{2} \operatorname{Tr} W\left(K_{T}^{0}(-i h \nabla)\right)^{-1} W \\
\quad \geq & \frac{1}{2} \operatorname{Tr} K_{T}^{0}(-i h \nabla)\left(\gamma-\gamma^{0}\right)^{2}-C h^{3} .
\end{aligned}
$$

The last step used that $\operatorname{Tr} W\left(K_{T}^{0}(-i h \nabla)\right)^{-1} W \leq\|W\|_{\infty}^{2} \operatorname{Tr} K_{T}^{0}(-i h \nabla)^{-1} \leq C h^{-1}$. 
Next, we treat the second line on the right side of (5.8). Recall that the BCS gap equation in the form (5.3) says that the operator $K_{T}^{0}(-i h \nabla)-a h \delta(x)$ has an eigenvalue zero with eigenfunction $\alpha^{0}(x / h)$. Hence

$$
\begin{aligned}
\operatorname{Tr} & K_{T}^{0}(-i h \nabla)\left(\alpha-\alpha^{0}\right)\left(\overline{\alpha-\alpha^{0}}\right)-a h \int_{\mathcal{C}}\left|\alpha(x, x)-\alpha^{0}(0)\right|^{2} d x \\
& =\operatorname{Tr} K_{T}^{0}(-i h \nabla) \alpha \bar{\alpha}-a h \int_{\mathcal{C}}|\alpha(x, x)|^{2} d x .
\end{aligned}
$$

Since a delta potential creates at most one bound state, zero must be the ground state energy of $K_{T}^{0}(-i h \nabla)-a h \delta(x)$, and we deduce that

$$
\operatorname{Tr} K_{T}^{0}(-i h \nabla) \alpha \bar{\alpha}-a h \int_{\mathcal{C}}|\alpha(x, x)|^{2} d x \geq 0 .
$$

This information, together with (5.9) and (5.8), yields

$$
\begin{gathered}
\operatorname{Tr}\left(1-h^{2} \nabla^{2}\right)\left(\gamma-\gamma^{0}\right)^{2} \leq C \operatorname{Tr} K_{T}^{0}(-i h \nabla)\left(\gamma-\gamma^{0}\right)^{2} \leq C h^{3}, \\
\operatorname{Tr} K_{T}^{0}(-i h \nabla) \alpha \bar{\alpha}-a h \int_{\mathcal{C}}|\alpha(x, x)|^{2} d x \leq C h^{3}
\end{gathered}
$$

and

$$
\frac{\left(\operatorname{Tr}\left[\gamma(1-\gamma)-\gamma^{0}\left(1-\gamma^{0}\right)-\alpha \bar{\alpha}+\alpha^{0} \overline{\alpha^{0}}\right]\right)^{2}}{\left|\operatorname{Tr}\left[\gamma(1-\gamma)-\gamma^{0}\left(1-\gamma^{0}\right)-\alpha \bar{\alpha}+\alpha^{0} \overline{\alpha^{0}}\right]\right|+\operatorname{Tr}\left[\gamma^{0}\left(1-\gamma^{0}\right)-\alpha^{0} \overline{\alpha^{0}}\right]} \leq C h^{3} .
$$

We know that $\operatorname{Tr}\left[\gamma^{0}\left(1-\gamma^{0}\right)-\alpha^{0} \overline{\alpha^{0}}\right] \leq C h^{-1}$ from the explicit solution in the translation invariant case, and therefore (5.12) yields

$$
\left|\operatorname{Tr}\left[\gamma(1-\gamma)-\gamma^{0}\left(1-\gamma^{0}\right)-\alpha \bar{\alpha}+\alpha^{0} \overline{\alpha^{0}}\right]\right| \leq C h .
$$

In order to derive from this an a priori estimate on $\alpha$ we use (5.10) and the Schwarz inequality to bound

$$
\left|\operatorname{Tr}\left(\gamma-\gamma^{0}\right)\right| \leq h^{-2} \operatorname{Tr} K_{T}^{0}(-i h \nabla)\left(\gamma-\gamma^{0}\right)^{2}+h^{2} \operatorname{Tr}\left(K_{T}^{0}(-i h \nabla)\right)^{-1} \leq C h
$$

and

$\left|\operatorname{Tr}\left(\gamma^{2}-\left(\gamma^{0}\right)^{2}\right)\right|=\left|\operatorname{Tr}\left(\gamma-\gamma^{0}\right)\left(\gamma+\gamma^{0}\right)\right| \leq h^{-2} \operatorname{Tr}\left(\gamma-\gamma^{0}\right)^{2}+h^{2} \operatorname{Tr}\left(\gamma+\gamma^{0}\right)^{2} \leq C h$.

Finally, since $\operatorname{Tr} \alpha^{0} \overline{\alpha^{0}} \leq C h$ (see (5.2)), we conclude from (5.13) that $\operatorname{Tr} \alpha \bar{\alpha} \leq C h$, as claimed.

5.3. Decomposition of $\alpha$. Here we quantify in which sense $\alpha(x, y)$ is close to $\frac{1}{2}(\psi(x)+\psi(y)) \alpha^{0}\left(h^{-1}(x-y)\right)$. There is one technical point that we would like to discuss before stating the result. The asymptotic form $\frac{1}{2}(\psi(x)+\psi(y)) \alpha^{0}\left(h^{-1}(x-\right.$ $y)$ ) will allow us in the next subsection to use the semiclassical results in a similar way as in the proof of the upper bound. Our semiclassics, however, require $\psi$ to be in $H^{2}$. While we naturally get an $H^{1}$ condition, the $H^{2}$ condition is achieved by introducing an additional parameter $\epsilon>0$, which will later chosen to go to zero as $h \rightarrow 0$. 
Proposition 2. Let $\Gamma$ be admissible with $\mathcal{F}^{\mathrm{BCS}}(\Gamma) \leq \mathcal{F}^{\mathrm{BCS}}\left(\Gamma^{0}\right)$. Then for every sufficiently small $\epsilon \geq h>0$, the operator $\alpha=[\Gamma]_{12}$ can be decomposed as

$$
\alpha(x, y)=\frac{1}{2}(\psi(x)+\psi(y)) \alpha^{0}\left(h^{-1}(x-y)\right)+\sigma(x, y)
$$

with a periodic function $\psi \in H^{2}(\mathcal{C})$ satisfying

$$
\|\psi\|_{H^{1}} \leq C, \quad\|\psi\|_{H^{2}} \leq C \epsilon h^{-1}
$$

and with

$$
\|\sigma\|_{H^{1}}^{2} \leq C \epsilon^{-2} h^{3} .
$$

More precisely, one has $\sigma=\sigma_{1}+\sigma_{2}$ with

$$
\left\|\sigma_{1}\right\|_{H^{1}}^{2} \leq C h^{3}
$$

and with $\sigma_{2}$ of the form

$$
\sigma_{2}(x, y)=\frac{1}{2}(\tilde{\psi}(x)+\tilde{\psi}(y)) \alpha^{0}\left(h^{-1}(x-y)\right),
$$

where the Fourier transform of $\tilde{\psi}$ supported in $\left\{|p| \geq \epsilon h^{-1}\right\}$. The Fourier transform of $\psi$ is supported in $\left\{|p|<\epsilon h^{-1}\right\}$.

We recall that the $H^{1}$ norm of an operator was introduced in (3.7).

Proof. Step 1. We can write (5.6) as

$$
0 \leq \int_{\mathcal{C}}\left\langle\alpha(\cdot, y)\left|K_{T}^{0}(-i h \nabla)-a h \delta(\cdot-y)\right| \alpha(\cdot, y)\right\rangle d y \leq C h^{3}
$$

Here, the operator $K_{T}^{0}(-i h \nabla)$ acts on the $x$ variable of $\alpha(x, y)$, and $\langle\cdot \mid \cdot\rangle$ denotes the standard inner product on $L^{2}(\mathbb{R})$.

Now we recall that the operator $K_{T}^{0}-a h \delta(\cdot-y)$ on $L^{2}(\mathbb{R})$ has a unique ground state, proportional to $\alpha^{0}\left(h^{-1}(\cdot-y)\right)$, with ground state energy zero. There are no further eigenvalues and the bottom of its essential spectrum is $\Delta_{0} / \tanh \left[\frac{\Delta_{0}}{2 T}\right] \geq 2 T$. In particular, there is a lower bound, independent of $h$, on the gap. We write

$$
\psi_{0}(y)=\left(h \int_{\mathbb{R}^{d}}\left|\alpha^{0}(x)\right|^{2} d x\right)^{-1} \int_{\mathbb{R}^{d}} \alpha^{0}\left(h^{-1}(x-y)\right) \alpha(x, y) d x
$$

and decompose

$$
\alpha(x, y)=\psi_{0}(y) \alpha^{0}\left(h^{-1}(x-y)\right)+\sigma_{0}(x, y) .
$$

Then (5.18) together with the uniform lower bound on the gap of $K_{T}^{0}-a h \delta(\cdot-y)$ yields the bound $\left\|\sigma_{0}\right\|_{2}^{2} \leq C h^{3}$. We can also symmetrize and write

$$
\sigma_{1}(x, y)=\sigma_{0}(x, y)+\frac{1}{2}(\psi(x)-\psi(y)) \alpha^{0}\left(h^{-1}(x-y)\right) .
$$

Then

again with

$$
\alpha(x, y)=\frac{1}{2}\left(\psi_{0}(x)+\psi_{0}(y)\right) \alpha^{0}\left(h^{-1}(x-y)\right)+\sigma_{1}(x, y)
$$

$$
\left\|\sigma_{1}\right\|_{2}^{2} \leq C h^{3} .
$$

This proves the first half of (5.17). Before proving the second half in Step 4 below we need to study $\psi$.

Step 2. We claim that

$$
\int_{\mathcal{C}}\left|\psi_{0}(x)\right|^{2} d x \leq C
$$


and

$$
\int_{\mathcal{C}}\left|\psi_{0}^{\prime}(x)\right|^{2} d x \leq C
$$

The first inequality follows by Schwarz's inequality

$$
\int_{\mathcal{C}}\left|\psi_{0}(x)\right|^{2} d x \leq \frac{\|\alpha\|_{2}^{2}}{h \int_{\mathbb{R}}\left|\alpha^{0}(x)\right|^{2} d x}
$$

and our bounds (5.5) and (5.2). In order to prove (5.24) we use again Schwarz's inequality,

$$
\int_{\mathcal{C}}\left|\psi_{0}^{\prime}(x)\right|^{2} d x \leq \frac{\int_{\mathbb{R} \times \mathcal{C}}\left|\left(\nabla_{x}+\nabla_{y}\right) \alpha(x, y)\right|^{2} d x d y}{h \int\left|\alpha^{0}(x)\right|^{2} d x} .
$$

Lemma 2 below bounds the numerator by a constant times

$$
h^{-2} \int_{\mathcal{C}}\left\langle\alpha(\cdot, y)\left|K_{T}^{0}(-i h \nabla)-a h \delta(\cdot-y)\right| \alpha(\cdot, y)\right\rangle d y,
$$

and therefore (5.24) is a consequence of (5.18) and (5.2).

Step 3. Next, we establish the remaining bound $\left\|\nabla \sigma_{1}\right\|_{2}^{2} \leq C h$ in (5.17). We use formula (5.20) for $\sigma_{1}$. First of all, using the fact that $K(-i h \nabla) \geq c\left(1-h^{2} \nabla^{2}\right)$ one easily deduces from (5.18) that $\left\|\nabla \sigma_{0}\right\|_{2}^{2} \leq C h$. Moreover, because of (5.2) and (5.24)

$$
\int_{\mathcal{C}}\left|\psi_{0}^{\prime}(x)\right|^{2} \int_{\mathbb{R}}\left|\alpha^{0}\left(h^{-1}(x-y)\right)\right|^{2} d x d y \leq C h
$$

Finally,

$$
\begin{aligned}
& h^{-2} \int_{\mathcal{C} \times \mathbb{R}}\left|\psi_{0}(x)-\psi_{0}(y)\right|^{2}\left|\left(\alpha^{0}\right)^{\prime}\left(h^{-1}(x-y)\right)\right|^{2} d x d y \\
& =h^{-1} 4 \sum_{p \in 2 \pi \mathbb{Z}}\left|\hat{\psi}_{0}(p)\right|^{2} \int_{\mathbb{R}}\left|\left(\alpha^{0}\right)^{\prime}(x)\right|^{2} \sin ^{2}\left(\frac{1}{2} h p x\right) d x \\
& \leq h \sum_{p \in 2 \pi \mathbb{Z}}|p|^{2}\left|\hat{\psi}_{0}(p)\right|^{2} \int_{\mathbb{R}}\left|\left(\alpha^{0}\right)^{\prime}(x)\right|^{2} x^{2} d x \leq C h,
\end{aligned}
$$

where we used (5.24) and the fact that $\int_{\mathbb{R}}\left|\left(\alpha^{0}\right)^{\prime}(x)\right|^{2} x^{2} d x$ is finite. This is a simple consequence of the fact that the Fourier transform of $\alpha^{0}$ is given by the smooth function $\frac{\Delta_{0}}{2(2 \pi)^{1 / 2}}\left(K_{T}^{0}\right)^{-1}$. This completes the proof of (5.17).

Step 4. Finally, for each $\epsilon \geq h$ we decompose $\psi_{0}=\psi+\tilde{\psi}$, where the Fourier transforms of $\psi$ and $\tilde{\psi}$ are supported in $\left\{|p|<\epsilon h^{-1}\right\}$ and $\left\{|p| \geq \epsilon h^{-1}\right\}$, respectively. Clearly, the bounds (5.23) and (5.24) imply (5.15).

Moreover, $\|\tilde{\psi}\|_{2} \leq C \epsilon^{-1} h$ and $\left\|\tilde{\psi}^{\prime}\right\|_{2} \leq C$, and hence

$$
\sigma_{2}(x, y)=\frac{1}{2}(\tilde{\psi}(x)+\tilde{\psi}(y)) \alpha^{0}\left(h^{-1}(x-y)\right)
$$

satisfies $\left\|\sigma_{2}\right\|_{H^{1}}^{2} \leq h^{3} \epsilon^{-2}$. This completes the proof of the proposition.

In the previous proof we made use of the following 
Lemma 2. For some constant $C>0$,

$$
\begin{aligned}
& h^{2} \int_{\mathbb{R} \times \mathcal{C}}\left|\left(\nabla_{x}+\nabla_{y}\right) \alpha(x, y)\right|^{2} d x d y \\
& \leq C \int_{\mathcal{C}}\left\langle\alpha(\cdot, y)\left|K_{T}^{0}(-i h \nabla)-a h \delta(\cdot-y)\right| \alpha(\cdot, y)\right\rangle d y
\end{aligned}
$$

for all periodic and symmetric $\alpha$ (i.e., $\alpha(x, y)=\alpha(y, x))$.

Proof. By expanding $\alpha(x, y)$ in a Fourier series

$$
\alpha(x, y)=\sum_{p \in 2 \pi \mathbb{Z}} e^{i p(x+y) / 2} \alpha_{p}(x-y)
$$

and using that $\alpha_{p}(x)=\alpha_{p}(-x)$ for all $p \in 2 \pi \mathbb{Z}$ we see that (5.26) is equivalent to

$$
K_{T}^{0}(-i h \nabla+h p / 2)+K_{T}^{0}(-i h \nabla-h p / 2)-2 a \delta(x / h) \geq \frac{2}{C} h^{2} p^{2}
$$

for all $p \in 2 \pi \mathbb{Z}$. This inequality holds for all $p \in \mathbb{R}$, in fact, for an appropriate choice of $C>0$, as we shall now show.

Since $K_{T}^{0} \geq \operatorname{const}\left(1+h^{2}(-i \nabla+p / 2)^{2}\right)$, it suffices to consider the case of $h p$ small. If $\kappa=\Delta_{0} / \tanh \left[\frac{\Delta_{0}}{2 T}\right] \geq 2 T$ denotes the gap in the spectrum of $K_{T}^{0}(-i h \nabla)-a h \delta$ above zero, and $h^{-1 / 2} \phi_{0}(x / h)$ its normalized ground state, proportional to $\alpha^{0}(x / h)$,

$$
\begin{aligned}
& K_{T}^{0}(-i h \nabla+h p / 2)+K_{T}^{0}(-i h \nabla-h p / 2)-2 a h \delta \\
& \geq \kappa\left[e^{i h x p / 2}\left(1-\left|\phi_{0}\right\rangle\left\langle\phi_{0}\right|\right) e^{-i h x p / 2}+e^{-i h x p / 2}\left(1-\left|\phi_{0}\right\rangle\left\langle\phi_{0}\right|\right) e^{i h x p / 2}\right] \\
& \geq \kappa\left[1-\left.\left|\int\right| \phi_{0}(x)\right|^{2} e^{-i h x p} d x \mid\right] .
\end{aligned}
$$

In order to see the last inequality, simply rewrite the term as $\kappa(2-|f\rangle\langle f|-| g\rangle\langle g|)$, where $|\langle f \mid g\rangle|^{2}=\left.\left|\int\right| \phi_{0}(x)\right|^{2} e^{-i h x p} d x \mid$, and compute the smallest eigenvalue of the corresponding $2 \times 2$ matrix. Since $\phi_{0}$ is reflection symmetric, normalized and satisfies $\int x^{2}\left|\phi_{0}\right|^{2} d x<\infty$ (see Step 4 in the proof of Proposition 2), we have

$$
1-\left.\left.\left|\int\right| \phi_{0}(x)\right|^{2} e^{-i h x p} d x\left|=\int\right| \phi_{0}(x)\right|^{2}(1-\cos (h p x)) d x \geq c h^{2} p^{2} .
$$

This completes the proof of (5.28).

5.4. The lower bound. Pick a $\Gamma$ with $\mathcal{F}^{\mathrm{BCS}}(\Gamma) \leq \mathcal{F}^{\mathrm{BCS}}\left(\Gamma^{0}\right)$ and let $\psi$ be as in Proposition 2 (depending on some parameter $\epsilon \geq h$ to be chosen later). As before we let $\Delta(x)=-\psi(x) \Delta_{0}$ and define $H_{\Delta}$ by (2.1). We also put $\Gamma_{\Delta}=\left(1+\exp \left(\beta H_{\Delta}\right)\right)^{-1}$.

Our starting point is the representation

$$
\begin{aligned}
\mathcal{F}^{\mathrm{BCS}}(\Gamma) & -\mathcal{F}^{\mathrm{BCS}}\left(\Gamma_{0}\right)=-\frac{T}{2} \operatorname{Tr}\left[\ln \left(1+e^{-\beta H_{\Delta}}\right)-\ln \left(1+e^{-\beta H_{0}}\right)\right] \\
& +\frac{T}{2} \mathcal{H}\left(\Gamma, \Gamma_{\Delta}\right)+\Delta_{0} \operatorname{Re} \int_{\mathcal{C}} \overline{\psi(x)} \alpha(x, x) d x-h a \int_{\mathcal{C}}|\alpha(x, x)|^{2} d x .
\end{aligned}
$$

(Compare with (5.7).) According to the decomposition (5.14) which, in view of the BCS gap equation (1.7), reads on the diagonal

$$
\alpha(x, x)=\psi(x) \alpha^{0}(0)+\sigma(x, x)=\frac{\Delta_{0} \psi(x)}{2 a h}+\sigma(x, x),
$$


we can obtain the lower bound

$$
\begin{aligned}
\mathcal{F}^{\mathrm{BCS}}(\Gamma)-\mathcal{F}^{\mathrm{BCS}}\left(\Gamma_{0}\right) \geq & -\frac{T}{2} \operatorname{Tr}\left[\ln \left(1+e^{-\beta H_{\Delta}}\right)-\ln \left(1+e^{-\beta H_{0}}\right)\right] \\
& +\frac{T}{2} \mathcal{H}\left(\Gamma, \Gamma_{\Delta}\right)-a h \int_{\mathcal{C}}|\sigma(x, x)|^{2} d x
\end{aligned}
$$

For the first two terms on the right side we apply the semiclassics from Theorem 2 Arguing as in the proof of the upper bound and taking into account the bounds on $\psi$ from Proposition 2 we obtain

$$
\begin{aligned}
\mathcal{F}^{\mathrm{BCS}}(\Gamma)-\mathcal{F}^{\mathrm{BCS}}\left(\Gamma_{0}\right) \geq & h^{3}\left(\mathcal{E}^{\mathrm{GL}}(\psi)-b_{3}\right)-C \epsilon^{2} h^{3} \\
& +\frac{T}{2} \mathcal{H}\left(\Gamma, \Gamma_{\Delta}\right)-a h \int_{\mathcal{C}}|\sigma(x, x)|^{2} d x .
\end{aligned}
$$

Our final task is to bound the last two terms from below. In the remainder of this subsection we shall show that

$$
\frac{T}{2} \mathcal{H}\left(\Gamma, \Gamma_{\Delta}\right)-a h \int_{\mathcal{C}}|\sigma(x, x)|^{2} d x \geq-C\left(\epsilon h^{3}+\epsilon^{-2} h^{4}\right) .
$$

The choice $\epsilon=h^{1 / 3}$ will then lead to

$$
\mathcal{F}^{\mathrm{BCS}}(\Gamma)-\mathcal{F}^{\mathrm{BCS}}\left(\Gamma_{0}\right) \geq h^{3}\left(E^{\mathrm{GL}}-b_{3}\right)-C h^{3+1 / 3},
$$

which is the claimed lower bound.

In order to prove (5.32) we again use the lower bound on the relative entropy from Lemma 1 to estimate

$$
T \mathcal{H}\left(\Gamma, \Gamma_{\Delta}\right) \geq \operatorname{Tr}\left[\frac{H_{\Delta}}{\tanh \frac{1}{2 T} H_{\Delta}}\left(\Gamma-\Gamma_{\Delta}\right)^{2}\right] .
$$

The next lemma will allow us to replace the operator $H_{\Delta}$ in this bound by $H_{0}$.

Lemma 3. There is a constant $c>0$ such that for all sufficiently small $h>0$

$$
\frac{H_{\Delta}}{\tanh \frac{1}{2 T} H_{\Delta}} \geq(1-c h) K_{T}^{0}(-i h \nabla) \otimes \mathbb{I}_{\mathbb{C}^{2}}
$$

Proof. An application of Schwarz's inequality yields that for every $0<\eta<1$

$$
H_{\Delta}^{2} \geq(1-\eta)\left(H_{\Delta_{0}}^{0}\right)^{2}-\eta^{-1}\left(\Delta_{0}^{2}\|\psi-1\|_{\infty}^{2}+h^{2}\|W\|_{\infty}^{2}\right) .
$$

The expansion formula [14, (4.3.91)]

$$
\frac{x}{\tanh (x / 2)}=2+\sum_{k=1}^{\infty}\left(2-\frac{2 k^{2} \pi^{2}}{x^{2} / 4+k^{2} \pi^{2}}\right)
$$

shows that $x \mapsto \sqrt{x} / \tanh \sqrt{x}$ is an operator monotone function. This operator monotonicity implies that

$$
\begin{aligned}
K_{T}^{0}(-i h \nabla) \otimes \mathbb{I}_{\mathbb{C}^{2}} & \leq \frac{(1-\eta)^{-1 / 2} \sqrt{H_{\Delta}^{2}+\eta^{-1}\left(\Delta_{0}^{2}\|\psi-1\|_{\infty}^{2}+h^{2}\|W\|_{\infty}^{2}\right)}}{\tanh \frac{1}{2 T}(1-\eta)^{-1 / 2} \sqrt{H_{\Delta}^{2}+\eta^{-1}\left(\Delta_{0}^{2}\|\psi-1\|_{\infty}^{2}+h^{2}\|W\|_{\infty}^{2}\right)}} \\
& \leq(1-\eta)^{-1 / 2} \frac{\sqrt{H_{\Delta}^{2}+\eta^{-1}\left(\Delta_{0}^{2}\|\psi-1\|_{\infty}^{2}+h^{2}\|W\|_{\infty}^{2}\right)}}{\tanh \frac{1}{2 T} \sqrt{H_{\Delta}^{2}+\eta^{-1}\left(\Delta_{0}^{2}\|\psi-1\|_{\infty}^{2}+h^{2}\|W\|_{\infty}^{2}\right)}} \\
& \leq(1-\eta)^{-1 / 2}\left(1+\frac{1}{4 T^{2} \eta}\left(\Delta_{0}^{2}\|\psi-1\|_{\infty}^{2}+h^{2}\|W\|_{\infty}^{2}\right)\right) \frac{H_{\Delta}}{\tanh \frac{1}{2 T} H_{\Delta}}
\end{aligned}
$$


for $0<\eta<1$. The Sobolev inequality and (5.15) show that $\|\psi\|_{\infty} \leq C\|\psi\|_{H^{1}} \leq C$, and hence the lemma follows by choosing $\eta=h$.

To proceed, we denote $\alpha_{\Delta}=\left[\Gamma_{\Delta}\right]_{12}$ and recall from Theorem 3 that

$$
\begin{aligned}
\alpha_{\Delta} & =\frac{\Delta_{0}}{4}\left(\psi K_{T}^{0}(-i h \nabla)^{-1}+K_{T}^{0}(-i h \nabla)^{-1} \psi\right)+\eta_{1}+\eta_{2} \\
& =\frac{1}{2}\left(\psi \alpha^{0}+\alpha^{0} \psi\right)+\eta_{1}+\eta_{2}
\end{aligned}
$$

with $\eta_{1}$ and $\eta_{2}$ satisfying the bounds (3.9) and (3.10). The second equality follows from the explicit form (1.10) of $\alpha^{0}$. Comparing this with (5.14) we infer that

$$
\alpha=\alpha_{\Delta}+\sigma-\eta_{1}-\eta_{2} .
$$

Then (5.33) and (5.34) imply that

$$
\begin{aligned}
\frac{T}{2} \mathcal{H}\left(\Gamma, \Gamma_{\Delta}\right)-a h \int_{\mathcal{C}}|\sigma(x, x)|^{2} d x \\
\geq(1-c h) \operatorname{Tr} K_{T}^{0}(-i h \nabla)\left(\alpha-\alpha_{\Delta}\right)\left(\overline{\alpha-\alpha_{\Delta}}\right)-a h \int_{\mathcal{C}}|\sigma(x, x)|^{2} d x \\
\geq(1-c h) \operatorname{Tr} K_{T}^{0}(-i h \nabla) \sigma \bar{\sigma}-a h \int_{\mathcal{C}}|\sigma(x, x)|^{2} d x \\
\quad-(1-c h) 2 \operatorname{Re} \operatorname{Tr} K_{T}^{0}(-i h \nabla) \sigma\left(\overline{\eta_{1}+\eta_{2}}\right) .
\end{aligned}
$$

In order to bound the first term on the right side from below we are going to choose a parameter $\rho \geq 0$ such that $c h+\rho \leq 1 / 2$. Here $c$ is the constant from (5.34). (Eventually, we will pick either $\rho=0$ or $\rho=1 / 4$, say.) Note that

$$
\begin{aligned}
(1-c h) \operatorname{Tr} K_{T}^{0}(-i h \nabla)-a h \delta=\rho K_{T}^{0}(-i h \nabla) & +(1-2 c h-2 \rho)\left(K_{T}^{0}(-i h \nabla)-a h \delta\right) \\
& +(c h+\rho)\left(K_{T}^{0}(-i h \nabla)-2 a h \delta\right) .
\end{aligned}
$$

We recall that the operator $K_{T}^{0}(-i h \nabla)-a h \delta$ is non-negative and that the operator $K_{T}^{0}(-i h \nabla)-2 a h \delta$ has a negative eigenvalue of order one (by the form boundedness of $\delta$ with respect to $\left.K_{T}^{0}(-i \nabla)\right)$. Hence $K_{T}^{0}(-i h \nabla)-2 a h \delta \geq-C_{1}$ with a constant $C_{1}$ independent of $h$. (In the following it will be somewhat important to keep track of various constants, therefore we introduce here a numbering.) Moreover, using the fact that $K_{T}^{0}(-i h \nabla) \geq c_{1}\left(1-h^{2} \nabla^{2}\right)$ we arrive at the lower bound

$$
(1-c h) \operatorname{Tr} K_{T}^{0}(-i h \nabla)-a h \delta \geq c_{1} \rho\left(1-h^{2} \nabla^{2}\right)-C_{1}(c h+\rho),
$$

which means for the first term on the right side of (5.36) that

$$
(1-c h) \operatorname{Tr} K_{T}^{0}(-i h \nabla) \sigma \bar{\sigma}-a h \int_{\mathcal{C}}|\sigma(x, x)|^{2} d x \geq c_{1} \rho\|\sigma\|_{H^{1}}^{2}-C_{1}(c h+\rho)\|\sigma\|_{2}^{2} .
$$

We now turn to the second term on the right side of (5.36). Theorem 3 together with the bounds (5.15) on $\psi$, implies that $\left\|\eta_{1}+\eta_{2}\right\|_{H^{1}}^{2} \leq C \epsilon^{2} h^{3}$. This bound, combined with $\|\sigma\|_{H^{1}}^{2} \leq C \epsilon^{-2} h^{3}$ from Lemma 2, however, is not good enough. (It leads to an error of order $h^{3}$.) Instead, we shall make use of the observation that in the decompositions $\sigma=\sigma_{1}+\sigma_{2}$ and $\eta_{1}+\eta_{2}$ one has

$$
\operatorname{Tr} K_{T}^{0}(-i h \nabla) \sigma_{2} \overline{\eta_{1}}=0 \text {. }
$$

This can be seen by writing out the trace in momentum space and recalling that the Fourier transform of the $\psi$ involved in $\sigma_{2}$ has support in $\left\{|p| \geq \epsilon h^{-1}\right\}$, whereas the one of the $\psi$ involved in $\eta_{1}$ has support in $\left\{|p|<\epsilon h^{-1}\right\}$ (see also (6.22) ). 
Using the estimates (5.17) and (3.10) on $\sigma_{1}$ and $\eta_{2}$ we conclude that

$$
\begin{aligned}
\left|\operatorname{Tr} K_{T}^{0}(-i h \nabla) \sigma\left(\overline{\eta_{1}+\eta_{2}}\right)\right| & \leq\left|\operatorname{Tr} K_{T}^{0}(-i h \nabla) \sigma_{1} \overline{\eta_{1}}\right|+\left|\operatorname{Tr} K_{T}^{0}(-i h \nabla) \sigma \overline{\eta_{2}}\right| \\
& \leq C_{2}\left(\epsilon h^{3}+h^{5 / 2}\|\sigma\|_{H^{1}}\right) .
\end{aligned}
$$

Combining (5.36), (5.37) and (5.38) we find that

$$
\begin{aligned}
& \frac{T}{2} \mathcal{H}\left(\Gamma, \Gamma_{\Delta}\right)-a h \int_{\mathcal{C}}|\sigma(x, x)|^{2} d x \\
& \quad \geq c_{1} \rho\|\sigma\|_{H^{1}}^{2}-C_{1}(c h+\rho)\|\sigma\|_{2}^{2}-2 C_{2}\left(\epsilon h^{3}+h^{5 / 2}\|\sigma\|_{H^{1}}\right) .
\end{aligned}
$$

Next, we are going to distinguish two cases, according to whether $4 C_{1}\|\sigma\|_{2}^{2} \leq$ $c_{1}\|\sigma\|_{H^{1}}^{2}$ or not. In the first case, we choose $\rho=1 / 4$ and $h$ so small that $c h+\rho \leq 1 / 2$. In this way we can bound the previous expression from below by

$$
\frac{1}{8} c_{1}\|\sigma\|_{H^{1}}^{2}-2 C_{2}\left(\epsilon h^{3}+h^{5 / 2}\|\sigma\|_{H^{1}}\right) \geq-8 c_{1}^{-1} C_{2}^{2} h^{5}-2 C_{2} \epsilon h^{3} .
$$

This proves the claimed (indeed, a better) bound (5.32) in this case.

Now assume, conversely, that $4 C_{1}\|\sigma\|_{2}^{2}>c_{1}\|\sigma\|_{H^{1}}^{2}$. Then we choose $\rho=0$ and bound (5.39) from below by

$$
\begin{aligned}
& -C_{1} \operatorname{ch}\|\sigma\|_{2}^{2}-2 C_{2}\left(\epsilon h^{3}+h^{5 / 2}\|\sigma\|_{H^{1}}\right) \\
& \quad \geq-C_{1} \operatorname{ch}\|\sigma\|_{2}^{2}-2 C_{2}\left(\epsilon h^{3}+h^{5 / 2}\left(4 C_{1} / c_{1}\right)^{1 / 2}\|\sigma\|_{2}\right) .
\end{aligned}
$$

The bound (5.16) on $\|\sigma\|_{2}$ now leads again to the claimed lower bound (5.32).

This concludes the proof of the lower bound to the free energy in Theorem 1 Concerning the statement about approximate minimizers we note that $\mathcal{F}^{\mathrm{BCS}}\left(\Gamma^{0}\right)-$ $\mathcal{F}^{\mathrm{BCS}}\left(\Gamma_{0}\right)=O\left(h^{3}\right)$ and that our a-priori bounds on $\alpha$ in Proposition 2 remain true under the weaker condition that $\mathcal{F}^{\mathrm{BCS}}(\Gamma) \leq \mathcal{F}^{\mathrm{BCS}}\left(\Gamma^{0}\right)+C h^{3}$. We leave the details to the reader.

\section{Proof of Semiclassical asymptotics}

In this section we shall sketch the proofs of Theorems 2 and 3 containing the semiclassical asymptotics. We shall skip some technical details and refer to [7 for a thorough discussion.

6.1. Preliminaries. It will be convenient to use the following abbreviations

$$
k=-h^{2} \nabla^{2}-\mu+h^{2} W(x), \quad k_{0}=-h^{2} \nabla^{2}-\mu .
$$

We will frequently have to bound various norms of the resolvents $(z-k)^{-1}$ for $z$ in the contour $\Gamma$ defined by $\operatorname{Im} z= \pm \pi /(2 \beta)$ for $\beta>0$. We state these auxiliary bounds separately.

For $p \geq 1$, we define the $p$-norm of a periodic operator $A$ by

$$
\|A\|_{p}=\left(\operatorname{Tr}|A|^{p}\right)^{1 / p}
$$

where Tr stands again for the trace per unit volume. We note that for a Fourier multiplier $A(-i h \nabla)$, these norms are given as

$$
\|A(-i h \nabla)\|_{p}=h^{-1 / p}\left(\int_{\mathbb{R}}|A(q)|^{p} \frac{d q}{2 \pi}\right)^{1 / p} .
$$

The usual operator norm will be denoted by $\|A\|_{\infty}$. 
Lemma 4. For $z=t \pm i \pi /(2 \beta)$ and all sufficiently small $h$ one has

$$
\left\|(z-k)^{-1}\right\|_{p} \leq C h^{-1 / p} \times\left\{\begin{array}{cl}
t^{-1 /(2 p)} & \text { for } t \gg 1 \\
|t|^{-1+1 /(2 p)} & \text { for } t \ll-1
\end{array} \quad \text { if } 1 \leq p \leq \infty,\right.
$$

as well as

$$
\left\|(z-k)^{-1}\right\|_{\infty} \leq C \times\left\{\begin{array}{cl}
1 & \text { for } t \gg 1 \\
|t|^{-1} & \text { for } t \ll-1
\end{array} .\right.
$$

Proof. The estimates are easily derived with $k_{0}$ instead of $k$ by evaluating the corresponding integral. Since the spectra of $k$ and $k_{0}$ agree up to $O\left(h^{2}\right)$ the same bounds hold for $k$.

6.2. Proof of Theorem 2, The function $f$ in (3.2) is analytic in the strip $|\operatorname{Im} z|<$ $\pi$, and we can write

$$
f\left(\beta H_{\Delta}\right)-f\left(\beta H_{0}\right)=\frac{1}{2 \pi i} \int_{\Gamma} f(\beta z)\left[\frac{1}{z-H_{\Delta}}-\frac{1}{z-H_{0}}\right] d z,
$$

where $\Gamma$ is the contour $z=r \pm i \frac{\pi}{2 \beta}, r \in \mathbb{R}$. We emphasize that this contour representation is not true for the operators $f\left(\beta H_{\Delta}\right)$ and $f\left(\beta H_{0}\right)$ separately (because of a contribution from infinity), but only for their difference.

We claim that

$$
\left[f\left(\beta H_{\Delta}\right)\right]_{11}=\overline{\left[f\left(\beta H_{\Delta}\right)\right]_{22}}-\beta \overline{\left[H_{\Delta}\right]_{22}} .
$$

Recall that $[\cdot]_{i j}$ denotes the $i j$ element of an operator-valued $2 \times 2$ matrix. To see (6.6), we introduce the unitary matrix

$$
U=\left(\begin{array}{cc}
0 & 1 \\
-1 & 0
\end{array}\right)
$$

and note that

$$
\left[f\left(\beta H_{\Delta}\right)\right]_{11}=-\left[U f\left(\beta H_{\Delta}\right) U\right]_{22} .
$$

On the other hand, $U H_{\Delta} U=-\overline{H_{\Delta}}$, which implies that

$$
U f\left(\beta H_{\Delta}\right) U=f\left(-\beta \overline{H_{\Delta}}\right)=\overline{f\left(-\beta H_{\Delta}\right)} .
$$

The claim (6.6) now follows from the fact that $f(-z)=f(z)-z$.

Subtracting (6.6) and the corresponding formula for $H_{0}$ and noting that $H_{\Delta}$ and $H_{0}$ coincide on the diagonal we find that the two diagonal entries of $f\left(\beta H_{\Delta}\right)-$ $f\left(\beta H_{0}\right)$ are complex conjugates of each other. Since their trace is real we conclude that

$$
\operatorname{Tr}\left[f\left(\beta H_{\Delta}\right)-f\left(\beta H_{0}\right)\right]=\frac{1}{\pi i} \int_{\Gamma} f(\beta z) \operatorname{Tr}\left[\frac{1}{z-H_{\Delta}}-\frac{1}{z-H_{0}}\right]_{11} d z .
$$

(For technical details concerning the interchange of the trace and the integral we refer to 7 .)

The resolvent identity and the fact that

$$
\delta:=H_{\Delta}-H_{0}=-h\left(\begin{array}{cc}
0 & \psi(x) \\
\psi(x) & 0
\end{array}\right)
$$


is off-diagonal (as an operator-valued $2 \times 2$ matrix) implies that

$$
\begin{aligned}
& \operatorname{Tr}\left[\frac{1}{z-H_{\Delta}}-\frac{1}{z-H_{0}}\right]_{11}=\operatorname{Tr}\left[\frac{1}{z-H_{0}}\left(\delta \frac{1}{z-H_{0}}\right)^{2}\right]_{11} \\
& \quad+\operatorname{Tr}\left[\frac{1}{z-H_{0}}\left(\delta \frac{1}{z-H_{0}}\right)^{4}\right]_{11}+\operatorname{Tr}\left[\frac{1}{z-H_{\Delta}}\left(\delta \frac{1}{z-H_{0}}\right)^{6}\right]_{11} \\
& =: I_{1}+I_{2}+I_{3} .
\end{aligned}
$$

In the following we shall prove that

$$
\begin{aligned}
\frac{1}{\pi i} \int_{\Gamma} f(\beta z) I_{1} d z= & -\frac{h \beta^{2}}{2}\|\psi\|_{2}^{2} \int_{\mathbb{R}} g_{0}\left(\beta\left(q^{2}-\mu\right)\right) \frac{d q}{2 \pi} \\
& +\frac{h^{3} \beta^{3}}{8}\left\|\psi^{\prime}\right\|_{2}^{2} \int_{\mathbb{R}}\left(g_{1}\left(\beta\left(q^{2}-\mu\right)\right)+2 \beta q^{2} g_{2}\left(\beta\left(q^{2}-\mu\right)\right)\right) \frac{d q}{2 \pi} \\
& +\frac{h^{3} \beta^{3}}{2}\langle\psi|W| \psi\rangle \int_{\mathbb{R}} g_{1}\left(\beta\left(q^{2}-\mu\right)\right) \frac{d q}{2 \pi} \\
& +O\left(h^{5}\right)\|\psi\|_{H^{2}}^{2} \\
\frac{1}{\pi i} \int_{\Gamma} f(\beta z) I_{2} d z= & \frac{h^{3} \beta^{3}}{8}\|\psi\|_{4}^{4} \int_{\mathbb{R}} \frac{g_{1}\left(\beta\left(q^{2}-\mu\right)\right)}{q^{2}-\mu} \frac{d q}{2 \pi}+O\left(h^{5}\right)\|\psi\|_{H^{1}}^{3}\|\psi\|_{H^{2}} \\
& \frac{1}{\pi i} \int_{\Gamma} f(\beta z) I_{3} d z=O\left(h^{5}\right)\|\psi\|_{H^{1}}^{6}
\end{aligned}
$$

and

This will clearly prove (3.6). We will treat the three terms $I_{3}, I_{2}$ and $I_{1}$ (in this order) separately.

$\mathbf{I}_{3}$ : With the notation $k$ introduced in (6.1) at the beginning of this section, we have

$$
I_{3}=\operatorname{Tr}\left[\frac{1}{z-H_{\Delta}}\right]_{11} \Delta \frac{1}{z+k} \Delta^{\dagger} \frac{1}{z-k} \Delta \frac{1}{z+k} \Delta^{\dagger} \frac{1}{z-k} \Delta \frac{1}{z+k} \Delta^{\dagger} \frac{1}{z-k} .
$$

Using Hölder's inequality for the trace per unit volume (see [7]) and the fact that $\left|z-H_{\Delta}\right| \geq \pi /(2 \beta)$, we get

$$
\left|I_{3}\right| \leq \frac{2 \beta}{\pi} h^{6}\|\psi\|_{\infty}^{6}\left\|(z-k)^{-1}\right\|_{6}^{3}\left\|(z+k)^{-1}\right\|_{6}^{3} .
$$

Together with (6.4), this yields

$$
\left|I_{3}\right| \leq \frac{C h^{5}}{1+|z|^{3}}\|\psi\|_{\infty}^{6} .
$$

Here it was important to get a decay faster than $|z|^{-2}$, since we need to integrate $I_{3}$ against the function $f$ which behaves linearly at $-\infty$. Since $\|\psi\|_{\infty} \leq C\|\psi\|_{H^{1}}$ by Sobolev inequalities we have completed the proof of (6.10).

$\mathbf{I}_{2}$ : We continue with

$$
I_{2}=\operatorname{Tr} \frac{1}{z-k} \Delta \frac{1}{z+k} \Delta^{\dagger} \frac{1}{z-k} \Delta \frac{1}{z+k} \Delta^{\dagger} \frac{1}{z-k} .
$$

By the resolvent identity we have

$$
\frac{1}{z-k}=\frac{1}{z-k_{0}}+\frac{1}{z-k_{0}} h^{2} W \frac{1}{z-k} .
$$


Using Hölder as above, we can bound

$$
\begin{aligned}
& \left|\operatorname{Tr}\left(\frac{1}{z-k}-\frac{1}{z-k_{0}}\right) \Delta \frac{1}{z+k} \Delta^{\dagger} \frac{1}{z-k} \Delta \frac{1}{z+k} \Delta^{\dagger} \frac{1}{z-k}\right| \\
& \quad \leq h^{6}\|W\|_{\infty}\left\|\left(z-k_{0}\right)^{-1}\right\|_{\infty}\|\psi\|_{\infty}^{4}\left\|(z-k)^{-1}\right\|_{3}^{3}\left\|(z+k)^{-1}\right\|_{\infty}^{2} .
\end{aligned}
$$

By (6.4) and (6.5) this is bounded by $C h^{5}\|\psi\|_{H^{1}(\mathcal{C})}^{4}\left(1+|z|^{5 / 2}\right)^{-1}$. What we effectively have achieved for this error is, therefore, to replace one factor of $(z-k)^{-1}$ in $I_{2}$ by a factor of $\left(z-k_{0}\right)^{-1}$

In exactly the same way we proceed with the remaining factors $(z-k)^{-1}$ and $(z+k)^{-1}$ in $I_{2}$. The only difference is that $k$ might now be replaced by $k_{0}$ in the terms we have already treated, but this does not effect the bounds.

The final result is that $(\pi i)^{-1} \int_{\Gamma} f(\beta z) I_{2} d z$ equals

$$
\frac{1}{\pi i} \int_{\Gamma} f(\beta z) \operatorname{Tr}\left[\frac{1}{z-k_{0}} \Delta \frac{1}{z+k_{0}} \Delta^{\dagger} \frac{1}{z-k_{0}} \Delta \frac{1}{z+k_{0}} \Delta^{\dagger} \frac{1}{z-k_{0}}\right] d z+O\left(h^{5}\right)\|\psi\|_{H^{1}}^{4},
$$

and it remains to compute the asymptotics of the integral.

Let us indicate how to perform the trace per unit volume $\operatorname{Tr}[\ldots]$. In terms of integrals the trace can be written as

$$
\begin{aligned}
\frac{h^{4}}{(2 \pi)^{4}} & \int_{0}^{1} d x_{1} \int_{\mathbb{R}} d x_{2} \int_{\mathbb{R}} d x_{3} \int_{\mathbb{R}} d x_{4} \int_{\mathbb{R}^{4}} d p_{1} d p_{2} d p_{3} d p_{4} \overline{\psi\left(x_{1}\right)} \psi\left(x_{2}\right) \overline{\psi\left(x_{3}\right)} \psi\left(x_{4}\right) \\
& \times \frac{e^{i p_{1}\left(x_{1}-x_{2}\right)}}{\left(z-\left(h^{2} p_{1}^{2}-\mu\right)\right)^{2}} \frac{e^{i p_{2}\left(x_{2}-x_{3}\right)}}{z+\left(h^{2} p_{2}^{2}-\mu\right)} \frac{e^{i p_{3}\left(x_{3}-x_{4}\right)}}{z-\left(h^{2} p_{3}^{2}-\mu\right)} \frac{e^{i p_{4}\left(x_{4}-x_{1}\right)}}{z+\left(h^{2} p_{4}^{2}-\mu\right)} .
\end{aligned}
$$

Since $\psi$ is periodic with period one we have

$$
\psi\left(x_{j}\right)=\sum_{l_{j} \in 2 \pi \mathbb{Z}} \hat{\psi}\left(l_{j}\right) e^{i x_{j} l_{j}}
$$

We insert this into the above integral and perform the integrals over $x_{2}, x_{3}, x_{4}$. This leads to $\delta$-distributions such that we can subsequently perform the integrals over $p_{2}, p_{3}, p_{4}$, as well as the integral over $x_{1}$. In this way we obtain

$$
\begin{aligned}
& \frac{1}{\pi i} \int_{\Gamma} f(\beta z) \operatorname{Tr}\left[\frac{1}{z-k_{0}} \Delta \frac{1}{z+k_{0}} \Delta^{\dagger} \frac{1}{z-k_{0}} \Delta \frac{1}{z+k_{0}} \Delta^{\dagger} \frac{1}{z-k_{0}}\right] d z \\
& \quad=h^{3} \sum_{p_{1}, p_{2}, p_{3} \in 2 \pi \mathbb{Z}} \widehat{\psi}\left(p_{1}\right) \widehat{\psi^{*}}\left(p_{2}\right) \widehat{\psi}\left(p_{3}\right) \widehat{\psi^{*}}\left(-p_{1}-p_{2}-p_{3}\right) F\left(h p_{1}, h p_{2}, h p_{3}\right)
\end{aligned}
$$

with

$$
\begin{aligned}
F\left(p_{1}, p_{2}, p_{3}\right)= & \frac{\beta^{4}}{\pi i} \int_{\Gamma} d z f(\beta z) \int_{\mathbb{R}} \frac{d q}{2 \pi} \frac{1}{\left(z-\beta\left(\left(q+p_{1}+p_{2}+p_{3}\right)^{2}+\mu\right)\right)^{2}} \\
& \times \frac{1}{z+\beta\left(\left(q+p_{1}+p_{2}\right)^{2}-\mu\right)} \frac{1}{z-\beta\left(\left(q+p_{1}\right)^{2}-\mu\right)} \frac{1}{z+\beta\left(q^{2}-\mu\right)} .
\end{aligned}
$$

The leading behavior is given by

$$
F(0,0,0) \sum_{p_{1}, p_{2}, p_{3} \in 2 \pi \mathbb{Z}} \widehat{\psi}\left(p_{1}\right) \widehat{\psi^{*}}\left(p_{2}\right) \widehat{\psi}\left(p_{3}\right) \widehat{\psi^{*}}\left(-p_{1}-p_{2}-p_{3}\right)=F(0,0,0)\|\psi\|_{4}^{4} .
$$

The integral $F(0,0,0)$ can be calculated explicitly and we obtain

$$
F(0,0,0)=\frac{\beta^{3}}{8} \int_{\mathbb{R}} \frac{g_{1}\left(\beta\left(q^{2}-\mu\right)\right)}{q^{2}-\mu} \frac{d q}{2 \pi}
$$


with $g_{1}$ from (3.4). In order to estimate the remainder we use the fact that [7]

$$
\left|F\left(p_{1}, p_{2}, p_{3}\right)-F(0,0,0)\right| \leq \text { const }\left(p_{1}^{2}+p_{2}^{2}+p_{3}^{2}\right) .
$$

Using Schwarz and Hölder we can bound

$$
\sum_{p_{1}, p_{2}, p_{3} \in 2 \pi \mathbb{Z}} p_{1}^{2}\left|\widehat{\psi}^{*}\left(p_{1}\right) \widehat{\psi}^{*}\left(p_{2}\right) \widehat{\psi}\left(p_{3}\right) \widehat{\psi}\left(-p_{1}-p_{2}-p_{3}\right)\right| \leq \mathrm{const}\|\psi\|_{H^{2}}\|\psi\|_{H^{1}}^{3}
$$

and equally with $p_{1}^{2}$ replaced by $p_{2}^{2}$ and $p_{3}^{2}$. Hence we conclude that

$$
\begin{aligned}
\frac{1}{\pi i} \int_{\Gamma} f(\beta z) I_{2} d z= & h^{3} \sum_{p_{1}, p_{2}, p_{3} \in 2 \pi \mathbb{Z}} \widehat{\psi}\left(p_{1}\right) \widehat{\psi^{*}}\left(p_{2}\right) \widehat{\psi}\left(p_{3}\right) \widehat{\psi^{*}}\left(-p_{1}-p_{2}-p_{3}\right) F\left(h p_{1}, h p_{2}, h p_{3}\right) \\
& +O\left(h^{5}\right)\|\psi\|_{H^{1}}^{4} \\
= & h^{3} F(0,0,0)\|\psi\|_{4}^{4}+O\left(h^{5}\right)\|\psi\|_{H^{1}}^{3}\|\psi\|_{H^{2}} .
\end{aligned}
$$

This is what we claimed in (6.9).

$\mathbf{I}_{\mathbf{1}}$ : Finally, we examine the contribution of

$$
I_{1}=\operatorname{Tr}\left[\frac{1}{z-k} \Delta \frac{1}{z+k} \Delta^{\dagger} \frac{1}{z-k}\right] .
$$

Using the resolvent identity (6.11) we can write $I_{1}=I_{1}^{a}+I_{1}^{b}+I_{1}^{c}$, where

$$
I_{1}^{a}=\operatorname{Tr}\left[\frac{1}{z-k_{0}} \Delta \frac{1}{z+k_{0}} \Delta^{\dagger} \frac{1}{z-k_{0}}\right]
$$

and

$$
\begin{aligned}
I_{1}^{b}=\operatorname{Tr}[ & \frac{1}{z-k_{0}}\left(k-k_{0}\right) \frac{1}{z-k_{0}} \Delta \frac{1}{z+k_{0}} \Delta^{\dagger} \frac{1}{z-k_{0}} \\
& +\frac{1}{z-k_{0}} \Delta \frac{1}{z+k_{0}}\left(k_{0}-k\right) \frac{1}{z+k_{0}} \Delta^{\dagger} \frac{1}{z-k_{0}} \\
& \left.+\frac{1}{z-k_{0}} \Delta \frac{1}{z+k_{0}} \Delta^{\dagger} \frac{1}{z-k_{0}}\left(k-k_{0}\right) \frac{1}{z-k_{0}}\right] .
\end{aligned}
$$

The part $I_{1}^{c}$ consists of the rest. We claim that

$$
\begin{aligned}
& \frac{1}{\pi i} \int_{\Gamma} f(\beta z) I_{1}^{a} d z=-\frac{h \beta^{2}}{2}\|\psi\|_{2}^{2} \int_{\mathbb{R}} g_{0}\left(\beta\left(q^{2}-\mu\right)\right) \frac{d q}{2 \pi} \\
&+\frac{h^{3} \beta^{3}}{8}\left\|\psi^{\prime}\right\|_{2}^{2} \int_{\mathbb{R}}\left(g_{1}\left(\beta\left(q^{2}-\mu\right)\right)+2 \beta q^{2} g_{2}\left(\beta\left(q^{2}-\mu\right)\right)\right) \frac{d q}{2 \pi} \\
&+O\left(h^{5}\right)\|\psi\|_{H^{2}}^{2} \\
& \frac{1}{\pi i} \int_{\Gamma} f(\beta z) I_{1}^{b} d z=\frac{h^{3} \beta^{3}}{2}\langle\psi|W| \psi\rangle \int_{\mathbb{R}} g_{1}\left(\beta\left(q^{2}-\mu\right)\right) \frac{d q}{2 \pi}+O\left(h^{5}\right)\|\psi\|_{H^{2}}\|\psi\|_{H^{1}}
\end{aligned}
$$

and

$$
\frac{1}{\pi i} \int_{\Gamma} f(\beta z) I_{1}^{c} d z=O\left(h^{5}\right)\|\psi\|_{H^{1}}^{2} .
$$

Clearly, this will imply (6.8).

We begin with $I_{1}^{c}$. These terms contain at least five resolvents, where at least two terms are of the form $\left(z-k_{\#}\right)^{-1}$ and at least one term of the form $\left(z+k_{\#}\right)^{-1}$. 
(Here $k_{\#}$ stands for any of the operators $k$ or $k_{0}$.) Moreover, they contain at least two factors of $k-k_{0}$. The terms are either of the type

$$
A=\operatorname{Tr} \frac{1}{z-k_{0}}\left(k-k_{0}\right) \frac{1}{z-k} \Delta \frac{1}{z+k_{0}}\left(k_{0}-k\right) \frac{1}{z+k} \Delta^{\dagger} \frac{1}{z-k_{0}}
$$

(at least three minus signs) or of the type

$$
B=\operatorname{Tr} \frac{1}{z-k_{0}} \Delta \frac{1}{z+k_{0}}\left(k_{0}-k\right) \frac{1}{z+k_{0}}\left(k_{0}-k\right) \frac{1}{z+k} \Delta^{\dagger} \frac{1}{z-k}
$$

(only two minus signs). Terms of the first type we bound by

$$
|A| \leq C h^{6}\|W\|_{\infty}^{2}\|\psi\|_{\infty}^{2}\left\|\left(z-k_{0}\right)^{-1}\right\|_{\infty}^{2}\left\|\left(z+k_{0}\right)^{-1}\right\|_{3}\left\|(z+k)^{-1}\right\|_{3}\left\|(z-k)^{-1}\right\|_{3} .
$$

By (6.4) and (6.5) this can be estimated by $C h^{5}|z|^{-2+1 / 6}$ if $\operatorname{Re} z \geq 1$ and by $C h^{5}|z|^{-2-1 / 6}$ if $\operatorname{Re} z \leq-1$. This bound is finite when integrated against $f(\beta z)$.

Terms of type $B$ can be bounded similarly by replacing $z$ by $-z$. Indeed, we note that since $\int_{\Gamma} z B d z=0$, we can replace $f(\beta z)$ by $f(-\beta z)=f(\beta z)-\beta z$ in the integrand without changing the value of the integral. I.e., we can integrate $B$ against a function that decays exponentially for negative $t$ and increases linearly for positive $t$, instead of the other way around. These considerations lead to the estimate (6.16).

Next, we discuss the term $I_{1}^{a}$. After doing the contour integral the term $I_{1}^{a}$ gives

$$
(\pi i)^{-1} \int_{\Gamma} f(\beta z) I_{1}^{a} d z=h \sum_{p \in 2 \pi \mathbb{Z}}|\hat{\psi}(p)|^{2} G(h p)
$$

with

$$
G(p)=-\frac{\beta}{2} \int_{\mathbb{R}} \frac{\tanh \left(\frac{1}{2} \beta\left((q+p)^{2}-\mu\right)\right)+\tanh \left(\frac{1}{2} \beta\left(q^{2}-\mu\right)\right)}{(p+q)^{2}+q^{2}-2 \mu} \frac{d q}{2 \pi} .
$$

By definition (3.3) we have

$$
G(0)=-\frac{\beta^{2}}{2} \int_{\mathbb{R}} g_{0}\left(\beta\left(q^{2}-\mu\right)\right) \frac{d q}{2 \pi} .
$$

Integrating by parts we can write

$$
G^{\prime \prime}(0)=\frac{\beta^{3}}{4} \int_{\mathbb{R}}\left(g_{1}\left(\beta\left(q^{2}-\mu\right)\right)+2 \beta q^{2} g_{2}\left(\beta\left(q^{2}-\mu\right)\right)\right) \frac{d q}{2 \pi}
$$

with $g_{1}$ and $g_{2}$ from (3.4) and (3.5). Moreover, one can show that 7

$$
\left|G(p)-G(0)-\frac{1}{2} p^{2} G^{\prime \prime}(0)\right| \leq C p^{4} .
$$

From this we conclude that

$$
\begin{aligned}
\frac{1}{\pi i} \int_{\Gamma} f(\beta z) I_{1}^{a} d z & =h \sum_{p \in 2 \pi \mathbb{Z}}|\hat{\psi}(p)|^{2}\left(G(0)+\frac{1}{2} G^{\prime \prime}(0) h^{2} p^{2}\right)+O\left(h^{5}\right)\|\psi\|_{H^{2}}^{2} \\
& =h G(0)\|\psi\|_{2}^{2}+\frac{1}{2} G^{\prime \prime}(0) h^{3}\left\|\psi^{\prime}\right\|_{2}^{2}+O\left(h^{5}\right)\|\psi\|_{H^{2}}^{2},
\end{aligned}
$$

which is what we claimed in (6.14).

Finally, we proceed to $I_{1}^{b}$. After the contour integration we find

$$
\frac{1}{\pi i} \int_{\Gamma} f(\beta z) I_{1}^{b} d z=h^{3} \sum_{p, q \in 2 \pi \mathbb{Z}} \widehat{\psi}^{*}(p) \widehat{\psi}(q) \widehat{W}(-p-q) L(h p, h q),
$$


where

$$
L(p, q)=\beta^{3} \int_{\mathbb{R}} L(p, q, k) \frac{d k}{2 \pi}
$$

with

$$
\begin{aligned}
L(p, q, k)=\frac{1}{\pi i} \int_{\Gamma} & \ln \left(2+e^{-\beta z}+e^{\beta z}\right) \frac{1}{z+k^{2}-\mu} \frac{1}{z-p^{2}+\mu} \frac{1}{z-q^{2}+\mu} \\
& \times\left(\frac{1}{z-p^{2}+\mu}+\frac{1}{z-q^{2}+\mu}+\frac{1}{z+k^{2}-\mu}\right) d z .
\end{aligned}
$$

We have

and (see [7] for details)

$$
L(0,0)=\frac{\beta^{3}}{2} \int_{\mathbb{R}} g_{1}\left(\beta\left(k^{2}-\mu\right)\right) \frac{d k}{2 \pi}
$$

$$
|L(p, q)-L(0,0)| \leq C\left(p^{2}+q^{2}\right) .
$$

By the Schwarz inequality we can bound

$$
\sum_{p, q \in 2 \pi \mathbb{Z}}\left|\widehat{\psi}^{*}(p) \widehat{\psi}(q) \widehat{W}(-p-q)\left(p^{2}+q^{2}\right)\right| \leq C\|W\|_{2}\|\psi\|_{H^{2}}\|\psi\|_{H^{1}},
$$

and obtain

$$
\begin{aligned}
\frac{1}{\pi i} \int_{\Gamma} f(\beta z) I_{1}^{b} d z & =h^{3} L(0,0) \sum_{p, q \in 2 \pi \mathbb{Z}} \widehat{\psi}^{*}(-p) \widehat{\psi}(q) \widehat{W}(p-q)+O\left(h^{5}\right)\|\psi\|_{H^{2}}\|\psi\|_{H^{1}} \\
& =\frac{h^{3} \beta^{3}}{2}\langle\psi|W| \psi\rangle \int_{\mathbb{R}} g_{1}\left(\beta\left(k^{2}-\mu\right)\right) \frac{d k}{2 \pi}+O\left(h^{5}\right)\|\psi\|_{H^{2}}\|\psi\|_{H^{1}}
\end{aligned}
$$

This concludes the proof of Theorem 2

6.3. Proof of Theorem [3. Since the function $\rho$ in (3.8) is analytic in the strip $|\operatorname{Im} z|<\pi$, we can write $\left[\rho\left(\beta H_{\Delta}\right)\right]_{12}$ with the aid of a contour integral representation as

$$
\left[\rho\left(\beta H_{\Delta}\right)\right]_{12}=\frac{1}{2 \pi i} \int_{\Gamma} \rho(\beta z)\left[\frac{1}{z-H_{\Delta}}\right]_{12} d z,
$$

where $\Gamma$ is again the contour $\operatorname{Im} z= \pm \pi /(2 \beta)$. We expand $\left(z-H_{\Delta}\right)^{-1}$ using the resolvent identity and note that, since $H_{\Delta}=H_{0}+\delta$ with a $H_{0}$ diagonal and $\delta$ offdiagonal, only the terms containing an odd number of $\delta$ 's contribute to the 12-entry of $\left(z-H_{\Delta}\right)^{-1}$. In this way arrive at the decomposition

$$
\left[\rho\left(\beta H_{\Delta}\right)\right]_{12}=\eta_{0}+\eta_{1}+\eta_{2}^{a}+\eta_{2}^{b}
$$

where

$$
\begin{gathered}
\eta_{0}=-\frac{h}{4 \pi i} \int_{\Gamma} \rho(\beta z)\left(\psi \frac{1}{z^{2}-k_{0}^{2}}+\frac{1}{z^{2}-k_{0}^{2}} \psi\right) d z \\
\eta_{1}=\frac{h}{4 \pi i} \int_{\Gamma} \rho(\beta z)\left(\frac{1}{z-k_{0}}\left[\psi, k_{0}\right] \frac{1}{z^{2}-k_{0}^{2}}+\frac{1}{z^{2}-k_{0}^{2}}\left[\psi, k_{0}\right] \frac{1}{z+k_{0}}\right) d z \\
\eta_{2}^{a}=-\frac{h^{3}}{2 \pi i} \int_{\Gamma} \rho(\beta z) \frac{1}{z-k_{0}}\left(W \frac{1}{z-k} \psi+\psi \frac{1}{z+k_{0}} W\right) \frac{1}{z+k} d z
\end{gathered}
$$

and

$$
\eta_{2}^{b}=-\frac{h^{3}}{2 \pi i} \int_{\Gamma} \rho(\beta z) \frac{1}{z-k} \psi \frac{1}{z+k} \bar{\psi} \frac{1}{z-k} \psi\left[\frac{1}{z-H_{\Delta}}\right]_{22} d z
$$


A simple residue computation yields

$$
\begin{aligned}
\eta_{0} & =-\frac{h}{4}\left(\psi \frac{\rho\left(\beta k_{0}\right)-\rho\left(-\beta k_{0}\right)}{k_{0}}+\frac{\rho\left(\beta k_{0}\right)-\rho\left(-\beta k_{0}\right)}{k_{0}} \psi\right) \\
& =\frac{h \beta}{4}\left(\psi g_{0}\left(\beta k_{0}\right)+g_{0}\left(\beta k_{0}\right) \psi\right),
\end{aligned}
$$

which is the main term claimed in the theorem. In the following we shall prove that

$$
\begin{aligned}
\left\|\eta_{1}\right\|_{H^{1}}^{2} & \leq C h^{5}\|\psi\|_{H^{2}}^{2}, \\
\left\|\eta_{2}^{a}\right\|_{H^{1}}^{2} & \leq C h^{5}\|\psi\|_{H^{1}}^{2},
\end{aligned}
$$

and

$$
\left\|\eta_{2}^{b}\right\|_{H^{1}}^{2} \leq C h^{5}\|\psi\|_{H^{1}}^{6}
$$

This clearly implies Theorem 3

$\eta_{1}$ : The square of the $H^{1}$ norm of $\eta_{1}$ is given by

$$
\left\|\eta_{1}\right\|_{H^{1}}^{2}=h \sum_{p \in 2 \pi \mathbb{Z}}|\hat{\psi}(p)|^{2} J(h p)
$$

with

$$
J(p)=\frac{\beta^{4}}{4} \int_{\mathbb{R}}\left((q+p)^{2}-q^{2}\right)^{2}\left(1+q^{2}\right)|F(q+p, q)-F(q, q+p)|^{2} \frac{d q}{2 \pi}
$$

and

$$
F(p, q)=\frac{1}{p^{2}-\mu} \frac{1}{1+e^{\beta\left(p^{2}-\mu\right)}} \frac{1}{1+e^{\beta\left(q^{2}-\mu\right)}}\left(\frac{e^{\beta\left(p^{2}-\mu\right)}-e^{\beta\left(q^{2}-\mu\right)}}{p^{2}-q^{2}}+\frac{e^{\beta\left(p^{2}+q^{2}-2 \mu\right)}-1}{p^{2}+q^{2}-2 \mu}\right) .
$$

One can show that $0 \leq J(p) \leq C p^{4}$ [7, which yields the desired bound (6.25).

$\eta_{\mathbf{2}}^{\mathbf{a}}$ : This term is a sum of two terms and we begin by bounding the first one, that is, $-h^{3}(2 \pi i)^{-1} \int \rho(\beta z)\left(z-k_{0}\right)^{-1} W(z-k)^{-1} \psi(z+k)^{-1} d z$. Using Hölder's inequality for the trace per unit volume we find that the square of the $H^{1}$ norm of the integrand can be bounded by

$$
\begin{aligned}
\operatorname{Tr} & {\left[\frac{1-h^{2} \nabla^{2}}{\left|z-k_{0}\right|^{2}} W \frac{1}{z-k} \psi \frac{1}{|z+k|^{2}} \bar{\psi} \frac{1}{\bar{z}-k} W\right] } \\
& \leq\left\|\frac{1-h^{2} \nabla^{2}}{\left|z-k_{0}\right|^{2}}\right\|_{\infty}\|W\|_{\infty}^{2}\|\psi\|_{\infty}^{2}\left\|(z-k)^{-1}\right\|_{\infty}^{2}\left\|(z+k)^{-1}\right\|_{2}^{2} .
\end{aligned}
$$

In order to bound this we use (6.4) and (6.5), as well as the fact that $\|\left(1-h^{2} \nabla^{2}\right) \mid z-$ $\left.k_{0}\right|^{-2} \|_{\infty}$ is bounded by $C|z|^{-1}$ if $\operatorname{Re} z \leq-1$ and by $C|z|$ if $\operatorname{Re} z \geq 1$. (This follows similarly as (6.5).) In particular, we conclude that for $\operatorname{Re} z \leq-1$ the previous quantity is bounded by $C h^{-1}\|\psi\|_{\infty}^{2}|z|^{-7 / 2}$. The square root of this is integrable against $\rho(\beta z)$ and we arrive at the bound $C h^{5 / 2}\|\psi\|_{\infty}$ for the $H^{1}$ norm. For the positive $z$ direction, we notice that $\rho(\beta z)$ decays exponentially leading to a finite result after $z$ integration.

For the second term in $\eta_{2}^{a}$ we proceed similarly. It is important to first notice that $\rho(z)=1-\rho(-z)$, however, and that the 1 does not contribute anything but integrates to zero. Proceeding as above we arrive at (6.26). 
$\eta_{\mathbf{2}}^{\mathbf{b}}$ : Finally, we consider $\eta_{2}^{b}$. Using Hölder's inequality for the trace per unit volume and bounding $\left[\left(z-H_{\Delta}\right)^{-1}\right]_{22}$ by $2 \beta / \pi$ for $z \in \Gamma$ we find that the square of the $H^{1}$ norm of the integrand is bounded by

$$
\frac{4 \beta^{2}}{\pi^{2}}\|\psi\|_{\infty}^{6}\left\|\frac{1-h^{2} \nabla^{2}}{\left|z-k_{0}\right|^{2}}\right\|_{\infty}\left\|(z-k)^{-1}\right\|_{\infty}^{2}\left\|(z+k)^{-1}\right\|_{2}^{2}
$$

Similarly as in the bound for $\eta_{2}^{a}$ one can show that for $\operatorname{Re} z \leq-1$ this is bounded by $C h^{-1}\|\psi\|_{\infty}^{6}|z|^{-7 / 2}$. This leads to (6.27).

Acknowledgments. Part of this work was carried out at the Erwin Schrödinger Institute for Mathematical Physics in Vienna, Austria, and the authors are grateful for the support and hospitality during their visit. Financial support via U.S. NSF grants DMS-0800906 (C.H.) and PHY-0845292 (R.S.) and a grant from the Danish council for independent research (J.P.S.) is gratefully acknowledged.

\section{REFERENCES}

[1] V.L. Ginzburg, L.D. Landau, Zh. Eksp. Teor. Fiz. 20, 1064 (1950).

[2] J. Bardeen, L. Cooper, J. Schrieffer, Theory of Superconductivity, Phys. Rev. 108, 1175-1204 (1957).

[3] L.P. Gor'kov, Zk, Eksperim. i Teor. Fiz. 36, 1918; 37, 833 (1959); Translation Soviet Phys. JETP 9,1364; 10, 593 (1960).

[4] P.G. de Gennes, Superconductivity of Metals and Alloys, Westview Press (1966).

[5] C. Hainzl, M. Lewin, R. Seiringer, A nonlinear theory for relativistic electrons at positive temperature, Rev. Math. Phys. 20, 1283-1307 (2008).

[6] R.L. Frank, C. Hainzl, S. Naboko, R. Seiringer, The critical temperature for the BCS equation at weak coupling, J. Geom. Anal. 17, 559-568 (2007).

[7] R. L. Frank, C. Hainzl, R. Seiringer, J. P. Solovej. Microscopic derivation of the GinzburgLandau theory, preprint, arXiv:1102.4001

[8] C. Hainzl, E. Hamza, R. Seiringer, J.P. Solovej, The BCS functional for general pair interactions, Commun. Math. Phys. 281, 349-367 (2008).

[9] C. Hainzl, R. Seiringer, Critical temperature and energy gap in the BCS equation, Phys. Rev. B 77, 184517 (2008).

[10] C. Hainzl, R. Seiringer, Spectral properties of the BCS gap equation of superfluidity. Mathematical results in quantum mechanics. Proceedings of the QMath10 conference, I. Beltita, G. Nenciu and R. Purice (eds.), World Scientific, 117-136 (2009)

[11] C. Hainzl, R. Seiringer, The BCS critical temperature for potentials with negative scattering length. Lett. Math. Phys. 84, No 2-3, 99-107 (2008)

[12] B. Helffer, D. Robert, Calcul fonctionnel par la transformation de Mellin et opérateurs admissibles, J. Funct. Anal. 53, 246-268 (1983).

[13] A.J. Leggett, Quantum Liquids, Oxford (2006).

[14] M. Abramowitz, I.A. Stegun, Handbook of mathematical functions, Dover (1964).

[15] W. Thirring, Quantum Mathematical Physics, $2^{\text {nd }}$ ed., Springer (2002).

[16] D. Robert, Autour de l'approximation semi-classique, Progress in Mathematics 68, Birkhäuser (1987). 
(Rupert L. Frank) Department of Mathematics, Princeton University, Princeton, NJ 08544, USA

E-mail address: rlfrank@math.princeton.edu

(Christian Hainzl) Mathematisches Institut, Universität Tübingen, Auf der MorgenStelle 10, 72076 TüBingen, Germany

E-mail address: christian.hainzl@uni-tuebingen.de

(Robert Seiringer) Department of Mathematics and Statistics, McGill University, 805 Sherbrooke Street West, Montreal, QC H3A 2K6, Canada

E-mail address: rseiring@math.mcgill.ca

(Jan Philip Solovej) Department of Mathematics, University of Copenhagen, UniverSitetsparken 5, DK-2100 Copenhagen, Denmark

E-mail address: solovej@math.ku.dk 